\title{
Impact of the Choice of Land Surface Scheme on a Simulated Heatwave Event: The Case of Sichuan-Chongqing Area, China
}

\author{
Y. Ma, ${ }^{1,2}$ X.-M. Zeng, ${ }^{2,3}$ Y. Zhang, ${ }^{4}$ N. Wang, ${ }^{2}$ Y. Zheng, ${ }^{2,3}$ G. Wang, ${ }^{1,3}$ and C. Chen ${ }^{1,3}$ \\ ${ }^{1}$ College of Meteorology and Oceanography, PLA University of Science and Technology, Nanjing, Jiangsu, China \\ ${ }^{2}$ College of Hydrology and Water Resources, Hohai University, Nanjing, Jiangsu, China \\ ${ }^{3}$ Key Laboratory for Mesoscale Severe Weather of Ministry of Education, Nanjing University, Nanjing, Jiangsu, China \\ ${ }^{4}$ Surveying, Mapping, and Meteorological Division, Unit 77200 of PLA, Kunming, China
}

Correspondence should be addressed to X.-M. Zeng; zen_xm@yahoo.com

Received 7 December 2016; Accepted 22 March 2017; Published 18 April 2017

Academic Editor: Pedro Jiménez-Guerrero

Copyright (C) 2017 Y. Ma et al. This is an open access article distributed under the Creative Commons Attribution License, which permits unrestricted use, distribution, and reproduction in any medium, provided the original work is properly cited.

\begin{abstract}
The Sichuan-Chongqing area in China has complex basin topography and is known for its extremely hot summer weather. In this paper, the mesoscale model WRF version 3.6.1 was used to simulate a period of 1-10 days in advance of the hot weather that occurred in Sichuan-Chongqing on August 10, 2006, to investigate the effect of six different land surface schemes (LSSs) (SLAB, RUC, PX, NOAH, NOAH_MP, and CLM4) on short- and medium-range simulations of high temperatures. The simulated surface air temperatures (SATs) are sensitive to the LSSs and simulation lengths. Specifically, all of the LSSs except PX generally reproduce the observed high temperatures, with CLM4 SATs at 06 UTC (SAT06) the most consistent with measurements whereas the shortrange (medium-range) results from NOAH_MP (NOAH) are the worst. Detailed explanations were given in terms of surface fluxes and physical processes. RUC soil moisture initialization appears poor and the LSS reflects too strong gravity drainage. When the LSSs with increased numbers of soil layers are used, the simulated high temperatures are found more consistent with measurements. Additionally, regional sensible heat flux (SHF) does not show high consistency with SAT. The results that differ from the previous studies are partly due to the complex geography and the LSS deficiencies.
\end{abstract}

\section{Introduction}

Over the past century, due to the combined effects of human activities and natural factors, Earth's climate has undergone a significant change in the primary features of warming, and this change has shown an increasing trend in recent years [1]. This trend has led to a wide range of climate anomalies and has especially caused the frequent occurrence of regional extreme high-temperature events. These events pose a serious threat to human survival, as well as social and economic development, water resources, and the ecological environment [2]. For example, in the summer of 2003, 35,000 people were killed by the heat wave in Europe [3]; during the summer of 2006, the most severe high temperatures and drought occurred in the Sichuan-Chongqing area in China since the beginning of the observational records [4].

Previous studies of high-temperature events have primarily focused on data analysis from the point of view of climate statistics or atmospheric circulation and have attempted to further explain how high temperatures occur. For example, Shi et al. [5] indicated that the average number of hightemperature days is 15 , and the average daily maximum temperature during those days achieved $36.3^{\circ} \mathrm{C}$ in East China over the past 46 years. Chen et al. [6] have shown that the combined Western Pacific Subtropical High (WPSH) and Mainland High $(\mathrm{MH})$ restrain the prevalent downdraft and convective activity in Sichuan-Chongqing, resulting in less rainfall, high temperatures, and drought in 2006.

In fact, there is a close relationship between high temperatures and the land surface. The land surface is composed of diverse and complex underlying surfaces, which are a very important part of the atmosphere boundary. The land surface process controls the exchange of momentum, energy, water vapor, and radiative transfer between the land surface and the atmosphere. It is one of the basic physical processes that affect local, regional, and global atmospheric circulation 
and climate change. Therefore, land surface processes have a very important impact on SATs, including high temperatures $[7,8]$. For example, Basara et al. [9] found urban areas were approximately $0.5^{\circ} \mathrm{C}$ warmer than the rural areas and over $2^{\circ} \mathrm{C}$ warmer at night during a heatwave that occurred in Oklahoma City at July and August 2008.

Because numerical models are an important tool for investigating mechanisms, several scholars have used coupled land-atmosphere models to discuss the influence of the land surface on heat wave simulation. For example, Wolfson et al. [10] conducted a series of medium-range experiments on American heat waves in the 1980s and found that abnormally high sea surface temperatures (SSTs) in the North Pacific are not conducive to maintaining heat waves, whereas abnormally low soil moisture (SM) values in the mainland of the United States are advantageous for maintaining heat waves. Fischer et al. [11] found that the intensity of heat waves during the summer of 2003 in Europe would be reduced by $40 \%$ if there were no reduction in early spring precipitation and SM. Zeng et al. [12] used the WRF model to perform 10 consecutive $24 \mathrm{~h}$ integrations covering East China during a short-term period of high temperatures in late July 2003 and indicated the existence of a negative feedback between circulation variations and SAT changes. Observations and simulation results obtained by Miralles et al. [13] revealed that the combined effects of dry soil and the accumulation of heat in the boundary layer at night can increase the occurrence probability of heat waves.

Reviewing previous work, we can see that these studies of high temperatures have focused mainly on their statistical characteristics or their theoretical causes, as well as numerical simulations over single time scales (either synoptic time scales or climatic time scales). At present, simulation studies that examine the sensitivity of high temperatures over shortand medium-range time scales to the land surface schemes (LSSs) in WRF are relatively rare. However, due to our focus on different weather forecast time scales, it is significant to compare the performance of the same model over both the short (e.g., [12]) and the medium (e.g., [10]) term. Therefore, Zeng et al. [14] simulated short- and medium-range high temperatures in East China using WRF 3.3 and found that the sensitivity of the simulated high temperatures to different LSSs over the medium term is greater than that over the short term. In addition, the change in high temperature is very consistent with the change in sensible heat flux (SHF); the SHF of the RUC (NOAH) scheme is the highest (lowest).

The differences in performance of these models are closely related to model complexity [15] and the characteristics of the area simulated [14]. Along with the development of the model system, the number of physical options has increased and the process descriptions are also more elaborate. For example, ARW-3.6.1 added CLM4 and NOAH_MP as LSS options. As the region in China that is prone to summer weather with high temperatures, the basins in Sichuan-Chongqing and low altitude areas characteristic of East China have great differences; the influence of the atmospheric system also shows obvious differences [6]. Therefore, there are some scientific problems in terms of the impact of the land-atmosphere coupling scheme in simulating high temperatures in Sichuan-Chongqing. For example, (1) what are the differences in performance between different LSSs in the simulation of high temperatures in the SichuanChongqing area? (2) What are the differences in the ability of a single LSS to simulate short- ( $\leq 2$ days) and medium- $(\geq 2$ days) term high temperatures? (3) What are the reasons for the above differences in the simulation of high temperatures? What regional characteristics exist?

To solve the above problems, we apply 6 LSSs in the mesoscale model WRF 3.6.1 and different simulation lengths (both short and medium range) to simulate high-temperature weather conditions on August 10, 2006, in the SichuanChongqing area. The structure of this paper is as follows. Section 2 describes the model and the experimental design; Section 3 describes the preliminary analysis of differences in the simulation results; Section 4 provides explanations of the simulated differences. Finally, we draw conclusions.

\section{Model and Experimental Design}

The model selected here is WRF 3.6.1; WRF is the nextgeneration mesoscale model developed by the National Centers for Environmental Prediction (NCEP) and other scientific research institutions [16]. The main physical schemes are similar to those presented in [12], and the potential LSSs that can be used in this model include the SLAB, RUC, PX, NOAH, NOAH_MP, and CLM4. SLAB [17] is transplanted from the corresponding scheme of the mesoscale model MM5, and its soil column is divided into 5 layers with thicknesses of $1,2,4,8$, and $16 \mathrm{~cm}$ without consideration of the effect of vegetation. RUC [18] solves for the soil temperature of six layers using the surface energy equations, which also include treatments of snow cover and the canopy. PX [19] consists of 2 layers of soil (a $1 \mathrm{~cm}$ thick surface layer and a $99 \mathrm{~cm}$ thick lower layer). It uses the force-restore method to calculate soil temperature and moisture, while accounting for the roles of vegetation and snow cover. NOAH [20] is developed at the Oregon State University (OSU) and is now used by many cooperating institutions. It can predict soil temperature and moisture in four layers with depths of 10, 30, 60 , and $100 \mathrm{~cm}$ with the forced recovery method.

NOAH_MP [21] is based on NOAH and was developed for use in ensembles in combination with a variety of parameterized schemes. The physical processes of NOAH's internal model have been improved greatly. In particular, the internal structure of the model, including its calculations of energy, water, and carbon fluxes, has been redesigned. At the same time, the canopy energy balance is separated from the surface energy balance and includes multiple layers of snow cover. Moreover, a groundwater factor and a runoff parameterization scheme have been added. The runoff scheme is based on terrain and accounts for the role of heterogeneities in SM in the control of runoff.

CLM4 [22] was developed by the National Center for Atmospheric Research (NCAR). It includes 10 soil layers and considers the differences in grid-scale surface features on the mainland and the effects of different types of vegetation on soil water dynamics and thermodynamics in different soil layers and implements these treatments through nested 




FIgure 1: Model domains (i.e., A1, A2, and A3), topography, and the distribution of observation stations (black dots).

subgrids. The biogeochemical module is based on ecological mechanisms and can be used to simulate carbon and nitrogen in vegetation, litter, and soil organic matter and simultaneously produces a water and energy balance forecast between vegetation, snow, and soil.

Using two-way nesting with two areas, the large area (A1) of the model is centered at $30^{\circ} \mathrm{N}, 106.5^{\circ} \mathrm{E}$, and includes $81 \times$ 64 grid points with a resolution of $30 \mathrm{~km}$. The small area (A2) has $100 \times 76$ grid points with a resolution of $10 \mathrm{~km}$ (Figure 1 ). Meanwhile, the terrain of the simulated area is shown in Figure 1. The elevations range from below $500 \mathrm{~m}$ within the Sichuan Basin to above $4000 \mathrm{~m}$ on the Tibetan Plateau, which demonstrates the complex geographical conditions.

The model features 31 uneven vertical levels, with the uppermost at a pressure of $50 \mathrm{hPa}$. The NCEP Final (FNL) reanalysis data with a $1^{\circ} \times 1^{\circ}$ resolution are used to specify the initial fields and boundary conditions in the simulations. To show the high-temperature characteristics of the Sichuan Basin area (A3) more accurately, if not specified, the analysis of the distribution of space below is based on the simulation results within $\mathrm{A} 2$, and the area-averaged physical quantities are based on simulation results from A3. Here, the end time of the simulations is 06 UTC August 10, 2006. Different LSSs were used to simulate the period starting 1 to 10 days ahead of this end time. According to these lead times, each experiment is named "DAY1" and "DAY2" till "DAY10". That is, simulations range from a $24 \mathrm{~h}$ long simulation beginning 1 day in advance and running from 06 UTC 9 August to 06 UTC 10 August, up to a $240 \mathrm{~h}$ long simulation beginning 10 days in advance and running from 06 UTC 31 July to 06 UTC 10 August. A total of $10 \times 6$ simulations were performed, and the time step for integration is $60 \mathrm{~s}$. It is worth noting is that such an experimental design can use the same evaluation standards (such as the SATs at 06 UTC 10 August), and this series of integration experiments, which have different simulation lengths, is reflective of not only this particular case but also the general characteristics of this type of short- and medium-range weather event.

The temperatures at 06 UTC are much closer to the maximum temperatures in the Sichuan-Chongqing area. Therefore, this paper analyzes the $2 \mathrm{~m}$ temperatures and related results at 06 UTC on August 10th.

\section{Preliminary Analysis of Simulation Differences}

3.1. Spatial Distribution of SAT. According to the characteristics of the climate and the environment in China, the daily extreme maximum temperatures were divided into three grades: high temperatures $\left(\geq 35^{\circ} \mathrm{C}\right)$, hazardous high temperatures $\left(\geq 38^{\circ} \mathrm{C}\right)$, and intensely hazardous high temperatures $\left(\geq 40^{\circ} \mathrm{C}\right)$ [12]. Because the physical parameter values and the data applied are the same, in addition to the LSSs and integration times used in each experiment, differences in the simulation results are caused by the different LSSs and different integration time lengths.

Because the initial and boundary conditions are the same, the simulated 06 UTC SAT (SAT06) values and the reanalysis data are consistent. At the same time, there are also large differences between the experiments, due to differences in the LSSs and simulation times used. Figure 2 takes the DAY1 experiments as an example to show the LSSs-simulated SAT06 distributions on 10 August and the corresponding FNL field. First, PX is largely unable to simulate the distribution of high temperatures (i.e., $\geq 35^{\circ} \mathrm{C}$ ) (Figure $2(\mathrm{k})$ ). In addition, it is still not able to simulate the high-temperature distributions over other time periods (i.e., the medium term) (figures omitted). This is very different from the previous simulation results (PX yields higher values in East China [14]). This result shows that, for the physical configuration scheme used in this paper, a parameterized algorithm like PX is completely unsuitable for the simulation of high temperatures in Sichuan-Chongqing. This is probably because the number of soil layers of PX is the least (i.e., 2 layers), which would lead to vertical-diffusion calculation with less precision [14]. Therefore, the analysis below focuses only on the remaining 5 LSSs not including PX.

For short-term simulations, such as the $24 \mathrm{~h}$ long DAY1 simulations, the 5 LSSs provide better simulations of the overall distribution of SAT06, in which high temperatures mainly appear within the Sichuan Basin, while lower temperatures occur in the surrounding areas; the average temperature is less than $27^{\circ} \mathrm{C}$ west of the basin. For the simulation of high temperatures, the 5 LSSs more accurately simulated the central position (approximately $30^{\circ} \mathrm{N}, 105^{\circ} \mathrm{E}$ ) and the overall distribution (Figures 2(a), 2(c), 2(e), 2(g), 2(i), and 2(l)). Meanwhile, it can be seen that the temperature distribution characteristics of the 5 LSSs are also very different. The simulated areas of high temperatures of CLM4 and RUC are more accurate, whereas the areas simulated using the other LSSs are obviously smaller. For hazardous high temperatures (i.e., $\geq 38^{\circ} \mathrm{C}$ ) and intensely hazardous high temperatures (i.e., $\geq 40^{\circ} \mathrm{C}$ ), CLM4 simulates the maximum area on the whole and has simulated a high-temperature center in the eastern part of area A2; however, the overall simulation results are the most accurate (Figure 2(i)). Although the center of the hightemperature region simulated by RUC is slightly south and the central intensity is slightly lower than FNL, it provides a more accurate simulation of the high-temperature zone (Figure 2(c)).

Compared to CLM4 and RUC, the other LSSs fail to simulate the hazardous high temperatures. The central position 


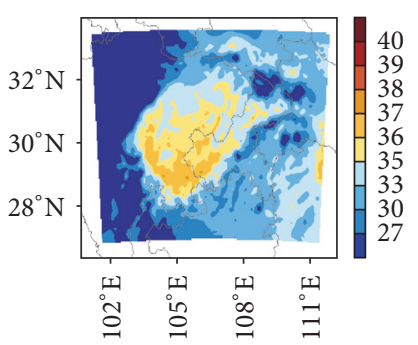

(a) SLAB (DAY1)

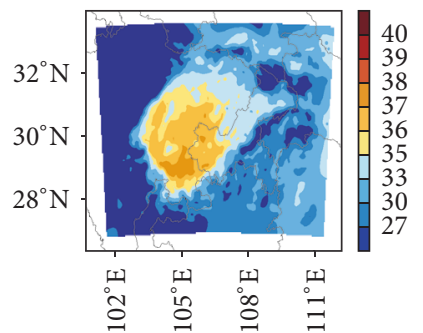

(e) NOAH (DAY1)

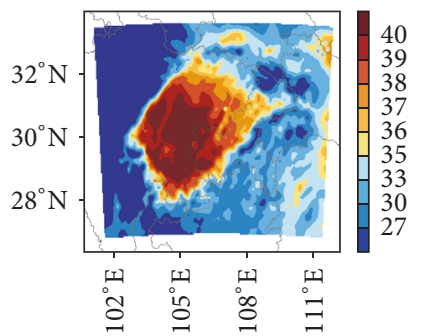

(i) CLM4 (DAY1)

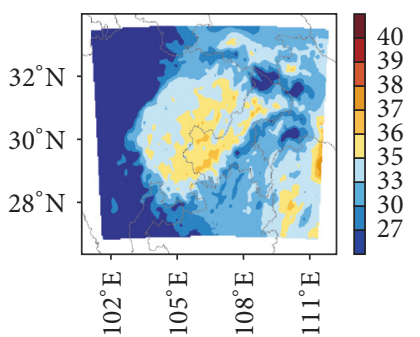

(b) SLAB (DAY9)

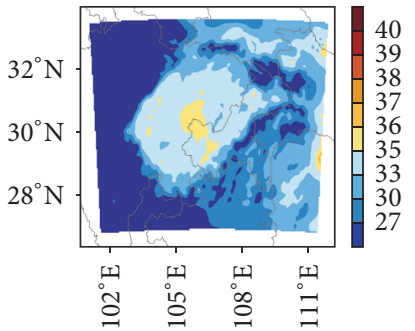

(f) NOAH (DAY10)

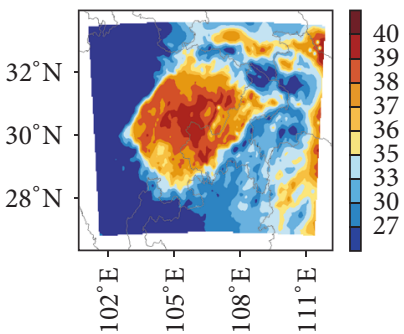

(j) CLM4 (DAY10)

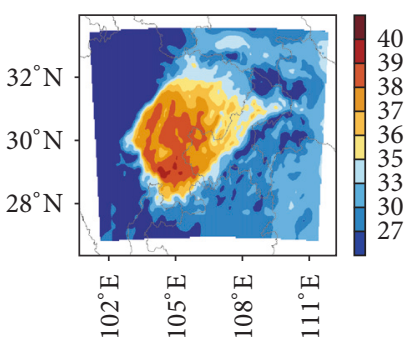

(c) RUC (DAY1)



(g) NOAH_MP (DAY1)



(k) PX (DAY1)

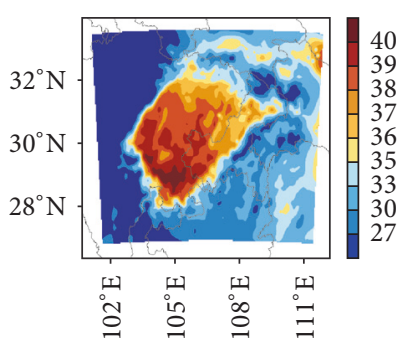

(d) RUC (DAY6)

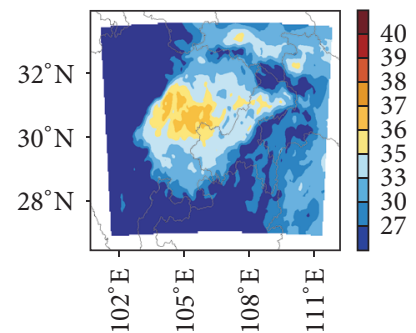

(h) NOAH_MP (DAY10)

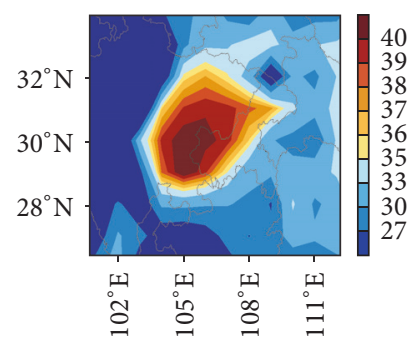

(l) FNL

Figure 2: Distributions of SAT06 (unit: ${ }^{\circ} \mathrm{C}$ ) on August 10, 2006, were (a) SLAB (DAY1), (b) SLAB (DAY9), (c) RUC (DAY1), (d) RUC (DAY6), (e) NOAH (DAY1), (f) NOAH (DAY10), (g) NOAH_MP (DAY1), (h) NOAH_MP (DAY10), (i) CLM4 (DAY1), (j) CLM4 (DAY10), (k) PX (DAY1), and (l) FNL.

simulated by NOAH_MP is more reasonable (Figure 2(g)), and the high-temperature distribution of NOAH is similar to that of NOAH_MP, but the central position of the high temperatures simulated by NOAH is farther south than when NOAH_MP is in use (Figure 2(e)). The SLAB-simulated hightemperature area is the minimum and its intensity is the lowest, but the SAT06 of the surrounding area outside the basin is generally higher than that of RUC (Figure 2(a)).

Figures $3(\mathrm{a})-3(\mathrm{~h})$ show the differences caused by different LSSs and simulation time lengths. In general, different LSSs exert considerable influence on the area and intensity of high temperatures in the short-term experiments. For example, the maximum single point difference of SAT06 in DAY1 can reach more than $5^{\circ} \mathrm{C}$ in CLM4-NOAH (Figure 3(f)).

In the medium-range simulations, the results from the 5 LSSs show their different variation characteristics compared to the short-term simulations. The following LSSs are analyzed one by one.

The SAT06 values simulated using SLAB have a very similar distribution before the DAY6 experiment and differ only in some southeastern parts of area A2, where they are gradually higher. The differences in the distribution of SAT06 between DAY1 and DAY5 show that they are concentrated within $1^{\circ} \mathrm{C}$ over most of the area (Figure 3(a)). However, after DAY6 and especially in DAY9 (Figure 2(b)) and DAY10 (figure omitted), the area and intensity of high temperatures decrease more obviously in the Sichuan-Chongqing area.

For the RUC, the area and intensity of high temperatures are essentially unchanged before DAY5. However, the areas with hazardous high temperatures increased significantly from DAY5 (figure omitted) to DAY6 (Figure 2(d)), especially for the intensely hazardous high temperatures. For the experiments with simulation lengths longer than DAY6, the SAT06 is slightly higher in the eastern part of area A2. In addition, the area and intensity of RUC-simulated high temperatures over the medium term are generally better than the results over the short term (e.g., DAY1-DAY10; Figure 3(b)).

The area and intensity of the high temperatures simulated by NOAH over the medium term are gradually reduced relative to the short term, but the temperatures in the eastern part of area $\mathrm{A} 2$ have risen slightly and are higher than the FNL data by approximately $1^{\circ} \mathrm{C}$. In DAY10 (Figure $2(f)$ ), the area of high temperatures is reduced to $1 / 5$ compared with the DAY1 experiment (Figure 2(e)) and the central intensity is reduced 


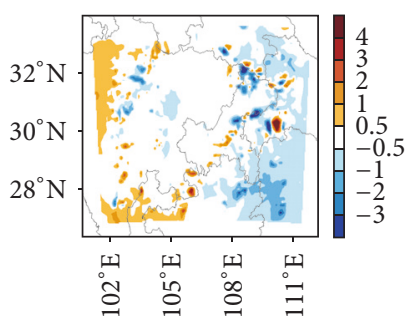

(a) DAY1-DAY5 (SLAB)



(e) DAY1-DAY10 (CLM4)



(b) DAY1-DAY10 (RUC)

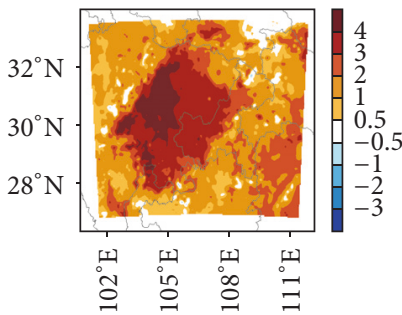

(f) CLM4-NOAH (DAY1)



(i) Diurnal SAT variation (DAY1)

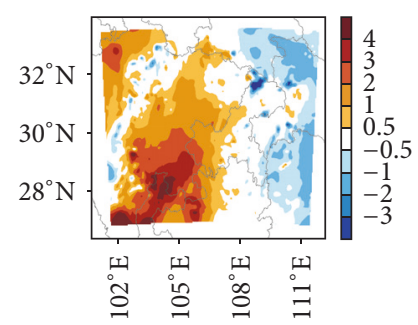

(c) DAY1-DAY10 (NOAH)

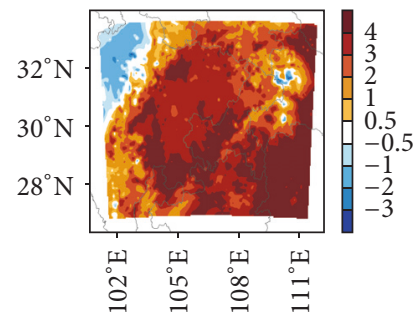

(g) CLM4-NOAH_MP (DAY4)

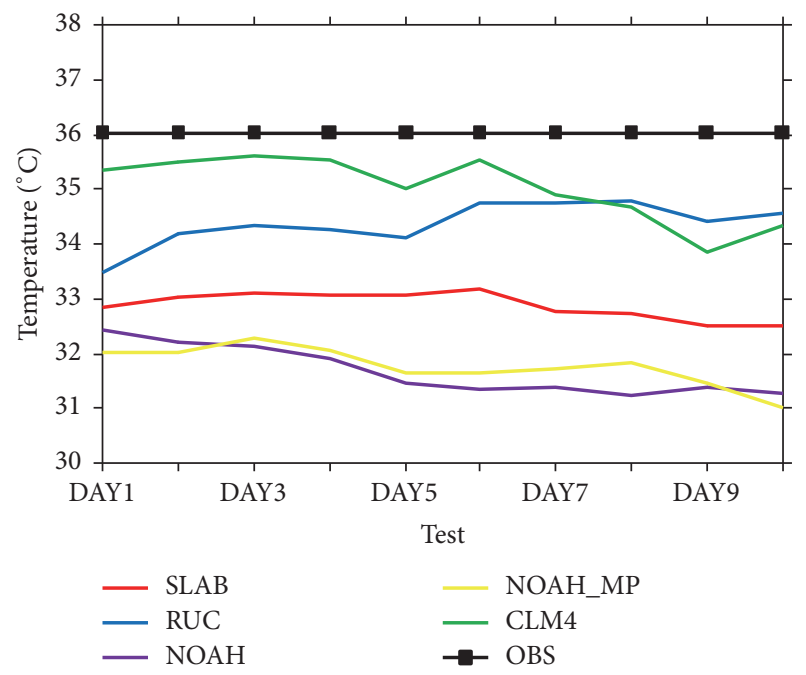

(j) SAT06 on August 10, 2006

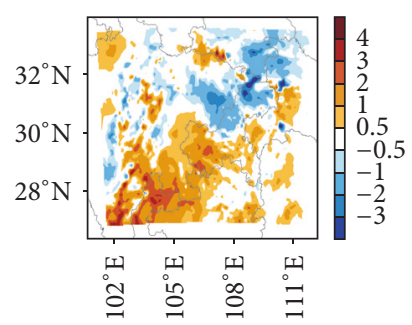

(d) DAY1-DAY8 (NOAH_MP)

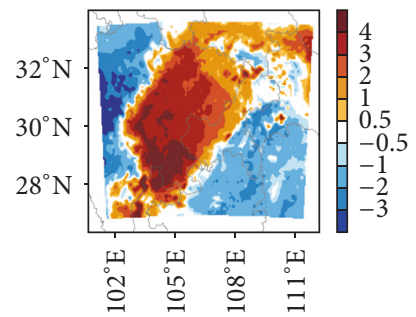

(h) RUC-SLAB (DAY8)

Figure 3: Distributions of SAT06 difference fields (unit: ${ }^{\circ} \mathrm{C}$ ) for August 10, 2006, due to different LSSs and simulation lengths (a-h) and the domain- (A3-) averaged DAY1 diurnal SAT variations (i) and SAT06 (j).

to $35^{\circ} \mathrm{C}$. Overall, $\mathrm{NOAH}$ is more sensitive to the simulation length of the simulation, and its simulation results become progressively worse (e.g., DAY1-DAY10; Figure 3(c)).

The SAT06 distribution simulated by NOAH_MP is more consistent before DAY8. For example, most of the differences in SAT06 between DAY1 and DAY8 are within $1^{\circ} \mathrm{C}$ (Figure $3(\mathrm{~d})$ ). In DAY10, the area of high temperatures significantly narrows and migrates to the northeast, while the intensity decreases from $37^{\circ} \mathrm{C}$ to $36^{\circ} \mathrm{C}$ (Figure 2(h)).

The CLM4-simulated SAT06 distribution is consistent from DAY1 to DAY4. The intensity significantly decreases in DAY5 and increases in DAY6. Finally, the intensity decreases progressively after DAY6, and the maximum temperature is reduced from $40^{\circ} \mathrm{C}$ to $39^{\circ} \mathrm{C}$ in DAY10 (Figure $2(\mathrm{j})$ ). The area of high temperatures on the east side of area A2 is gradually increasing in the short-term experiments and remains basically unchanged afterward. All in all, the
CLM4-simulated high temperatures are sensitive to the simulation length, and the intensity differs greatly between the short and medium term (e.g., DAY1-DAY10; Figure 3(e)).

Thus, great differences are induced by the 5 LSSs in the simulated area and intensity of high temperatures over the medium term. From this perspective, the biggest differences occur between CLM4 and NOAH_MP (e.g., the SAT06 differences in DAY4 in area A2 nearly exceed $4^{\circ} \mathrm{C}$; Figure 3(g)) and between RUC and SLAB (e.g., the SAT06 differences in DAY8 in the local region reached $5^{\circ} \mathrm{C}$ or higher; Figure 3(h)). The smallest differences are between $\mathrm{NOAH}$ and NOAH_MP (figure omitted). In summary, the simulated high temperatures are sensitive to the different LSSs and simulation lengths.

3.2. Area-Averaged SATs. This paper focuses on the sensitivity of high-temperature weather simulations to the LSSs in the 
Sichuan-Chongqing area. To avoid the errors induced by the surrounding mountains with low temperatures in area A2, area A3 was used to calculate area-averaged physical quantities (Figure 1).

Figure 3(i) uses the DAY1 experiments as an example to show the hourly variations in the SATs simulated by the 5 LSSs as averaged over area A3. It can be seen that all the LSSs are in accord with the temperature change rule. For example, the lowest daily SATs appear at approximately 22 UTC, and the highest daily SATs occur at approximately 06 UTC. The variations and magnitudes of SATs simulated by NOAH, SLAB, and NOAH_MP are consistent. The RUC-simulated SATs from $11 \mathrm{~h}$ to $23 \mathrm{~h}$ are still the highest, especially at $12 \mathrm{~h}$ and $13 \mathrm{~h}$, which are higher than those produced by the other LSSs by approximately $3^{\circ} \mathrm{C}$. The SATs produced by CLM 4 are the highest after $23 \mathrm{~h}$.

In addition, Figure $3(\mathrm{j})$ shows the variations in areaaveraged SATs due to the different simulation lengths used. All of the simulated SATs are lower than the measured value $\left(36^{\circ} \mathrm{C}\right)$. The CLM4-simulated SATs are the closest to the measured value and gradually decrease over the medium term. RUC-simulated SATs increase gradually and generally remain above $34^{\circ} \mathrm{C}$. Specifically, SAT06 increases by $1^{\circ} \mathrm{C}$ from DAY1 to DAY10. For SLAB, the SATs are basically stable at values below $33^{\circ} \mathrm{C}$ and the SATs show a slight decrease after DAY6, which shows the SLAB has good consistency between short- and medium-range simulations. On the other hand, NOAH and NOAH_MP-simulated SATs show a downward trend and decline by about $1^{\circ} \mathrm{C}$ with simulation lengths between DAY1 and DAY10.

On the whole, as reported by [14], there are systematic differences between the different LSSs. However, in contrast with the order of RUC, PX, SLAB, and NOAH that was found in East China, the simulated SATs generally decline in accordance with the order of CLM4, RUC, SLAB, and NOAH_MP in the Sichuan-Chongqing area. At the same time, NOAH and NOAH_MP are almost equivalent. In addition, the biggest systematic differences can reach approximately $3.6^{\circ} \mathrm{C}$ (i.e., the SAT06 differences between CLM4 and NOAH), which further demonstrates the significance of LSSs for the simulation of high temperatures.

\subsection{Evaluation of Simulated Results}

3.3.1. Student's $t$-Test. To further illustrate the sensitivity of the simulation of high temperatures to LSSs, Figure 4 shows the differences between different LSSs in terms of the mean of 10 experiments and Student's $t$-test results for August 10. In terms of differences in the spatial distribution (Figures 4(a)-4(d)), SLAB-simulated SAT06 values are basically similar or lower in the area of high temperatures (i.e., the Sichuan Basin) and higher outside the Sichuan Basin compared with other LSSs. Among them, the SAT06 differences of CLM4SLAB are the greatest at $4^{\circ} \mathrm{C}$ (Figure $4(\mathrm{~d})$ ), and the differences of NOAH-SLAB are the smallest, with values less than $-1^{\circ} \mathrm{C}$ (Figure 4(b)) in Sichuan Basin. Relative to RUC, the NOAHand NOAH_MP-simulated SAT06 values are generally lower (Figures 4(e) and 4(f)) and the CLM4-simulated SAT06 values are lower in the southwest part of area $\mathrm{A} 2$, whereas the portion of the A2 area with its southwestern part excluded is higher (Figure 4(g)). The SAT06 differences of NOAH_MP$\mathrm{NOAH}$ were mostly concentrated within $1^{\circ} \mathrm{C}$, which are positive in the northwestern part of area $\mathrm{A} 2$ and are negative in the southeastern part of area A2 (Figure 4(h)). The differences of CLM4-NOAH and CLM4-NOAH_MP are all above $2^{\circ} \mathrm{C}$ over the whole area (A2) (Figures 4(i) and 4(j)).

In addition, we can see that the SAT06 differences between different LSSs can reach a 5\% significance level in the Sichuan-Chongqing area using Student's $t$-test, which further confirms that the simulation of high temperatures has a very high sensitivity to the LSS used. However, these results do contain differences. For example, the significant areas of CLM4-RUC and NOAH_MP-NOAH are the smallest (Figures 4(g) and 4(h)), while the significant areas of CLM4NOAH and CLM4-NOAH_MP are the biggest, basically covering the entire area (A2) (Figures 4(i) and 4(j)). We can clearly see that the Sichuan Basin is an area of large differences in SAT06 from the spatial distribution differences among all the LSSs mentioned above.

3.3.2. Threat Score. The threat score (TS) (Figure 5(a)) is used to test the accuracy of LSS-simulated high temperatures, as described by [14].

For the different LSSs with the same simulation times, the TS values simulated by CLM4, RUC, SLAB, NOAH_MP, and NOAH decrease in turn. Meanwhile, the differences among the TS values from medium-range simulations are larger than those from short-term simulations. Comparison of the results in Figure 3(j) shows that the TS values and the SAT06 levels display very good consistency. That is, the higher the SAT06 value is, the higher the TS value will be. In the short-term simulations, the maximum TS difference is 0.35 in DAY2 (CLM4-NOAH_MP), while in the mediumrange simulations, the maximum TS difference is 0.50 in DAY7 (CLM4-NOAH).

For the same LSS, the TS values also show great differences with different simulation lengths. The TS values simulated by CLM4 increase from DAY1 (0.54) to DAY4 (0.68) and decrease from DAY4 to DAY10 (0.61). The TS values of RUC are slightly lower than those of CLM4, and the change is small. The TS values of SLAB show similar changes to those of CLM4, and the highest (lowest) TS value is 0.51 (0.34) during DAY6 (DAY1). The TS of NOAH displays the biggest changes with simulation length; it shifts from 0.31 during DAY1, when it is the highest, down to 0.11 on DAY10, when it is the lowest, and the relative magnitude of the fall is $170 \%$ of the lowest score.

3.3.3. Error Analysis. Neither measured nor simulated high temperatures (i.e., $\leq 35^{\circ} \mathrm{C}$ ) at stations in area $\mathrm{A} 2$ were removed, in order to accurately calculate the errors of the simulated high temperatures. We apply the model bias (BIAS; Figure 5(b)) and root mean squared error (RMSE; Figure 5(c)) to perform an error analysis, following [14].

The BIAS shows that the SAT06 values simulated using the 5 LSSs are all lower than the observed values. Combined with the TS values (Figure 5(a)), we can see that the larger overall errors associated with the LSSs correspond to lower 




(a) RUC-SLAB

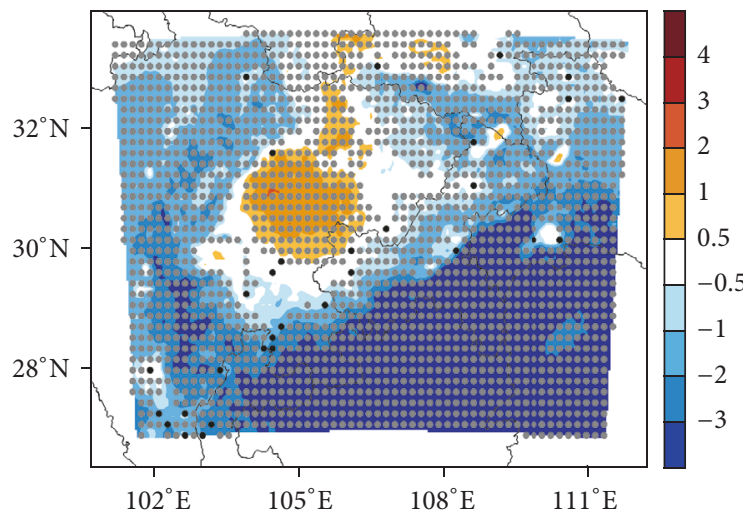

(c) NOAH_MP-SLAB



$102^{\circ} \mathrm{E} \quad 105^{\circ} \mathrm{E} \quad 108^{\circ} \mathrm{E} \quad 111^{\circ} \mathrm{E}$

(e) NOAH-RUC

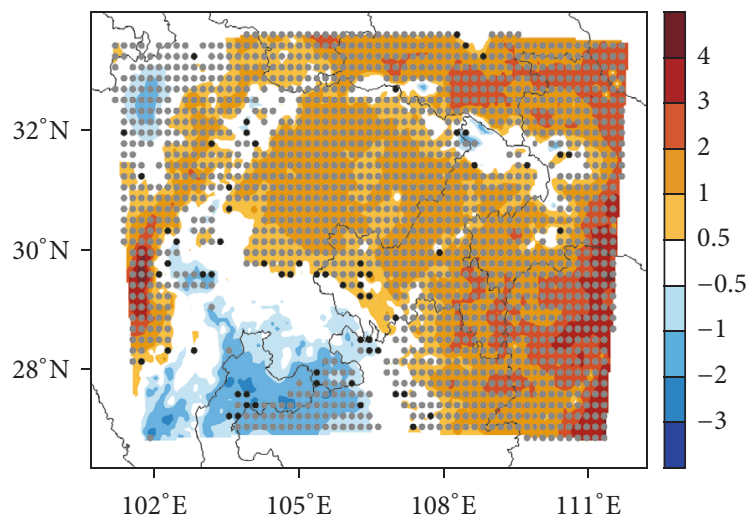

(g) CLM4-RUC

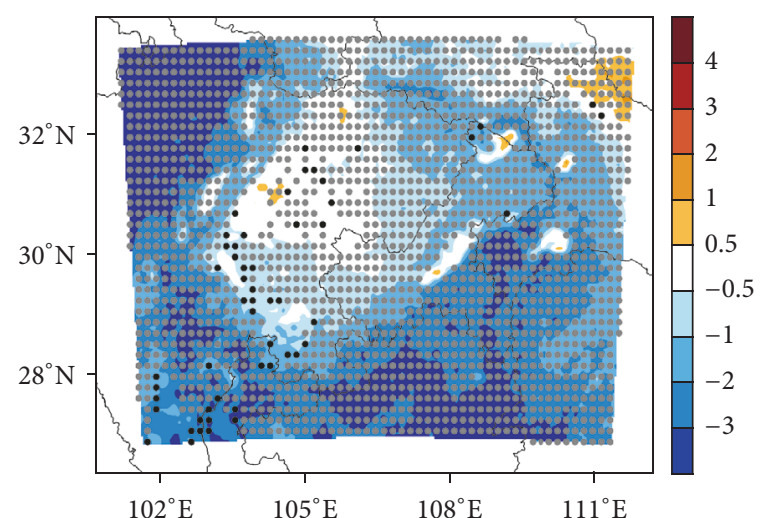

(b) NOAH-SLAB



(d) CLM4-SLAB



$102^{\circ} \mathrm{E} \quad 105^{\circ} \mathrm{E} \quad 108^{\circ} \mathrm{E} \quad 111^{\circ} \mathrm{E}$

(f) NOAH_MP-RUC

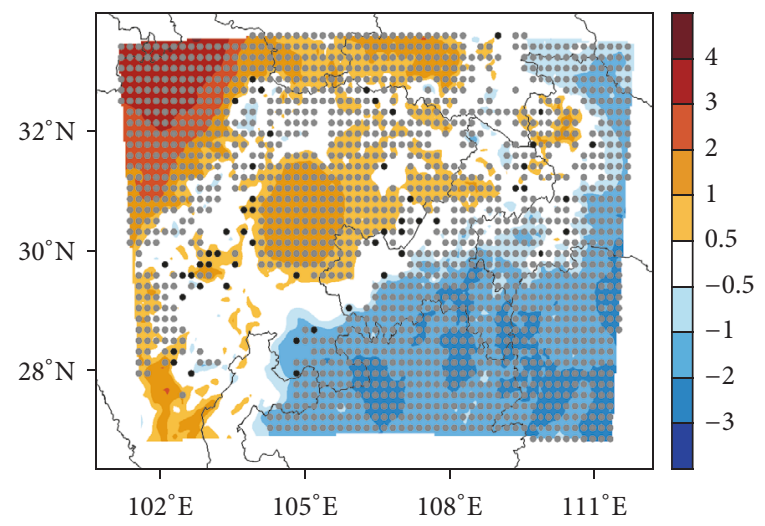

(h) NOAH_MP-NOAH

FIgUre 4: Continued. 


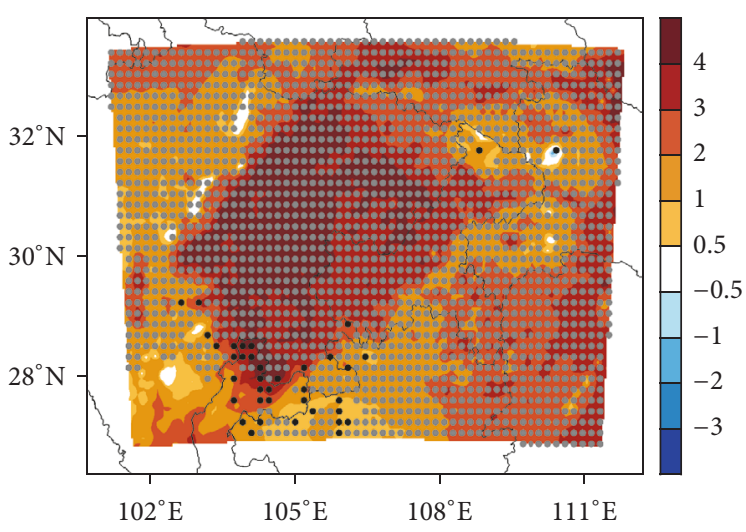

(i) CLM4-NOAH

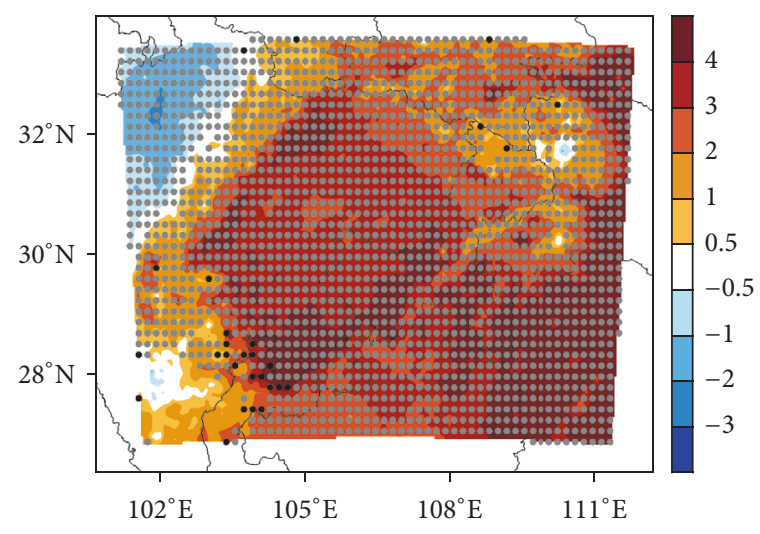

(j) CLM4-NOAH_MP

Figure 4: Mean SAT06 differences from 10 experiments (unit: ${ }^{\circ} \mathrm{C}$ ) and the results of Student's $t$-test for August 10 as simulated by different LSSs (gray and gray plus black dots denote grid points at significance levels of $5 \%$ and $10 \%$, resp.).

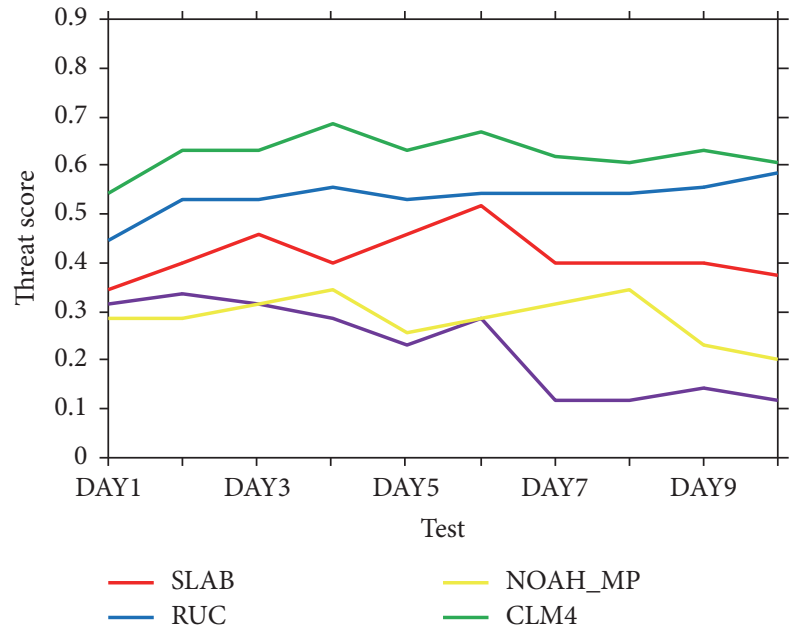

(a)

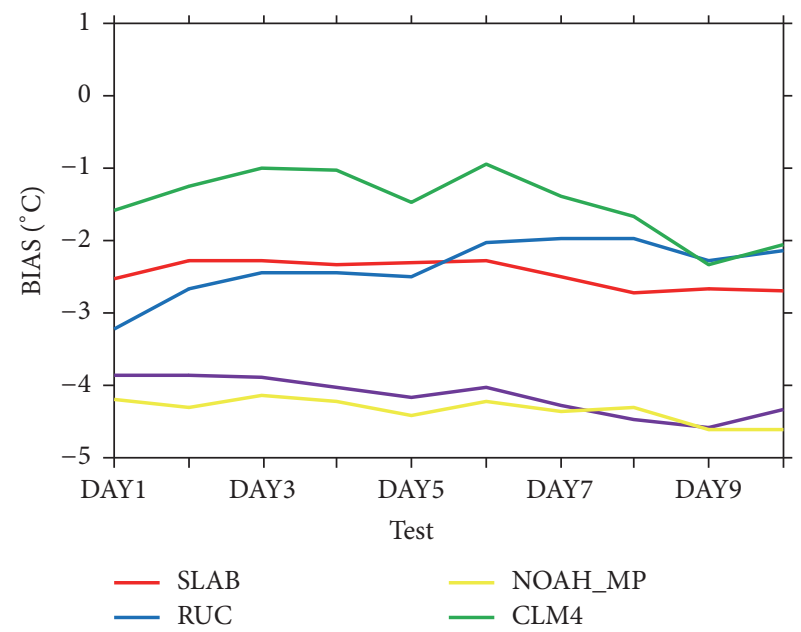

(b)

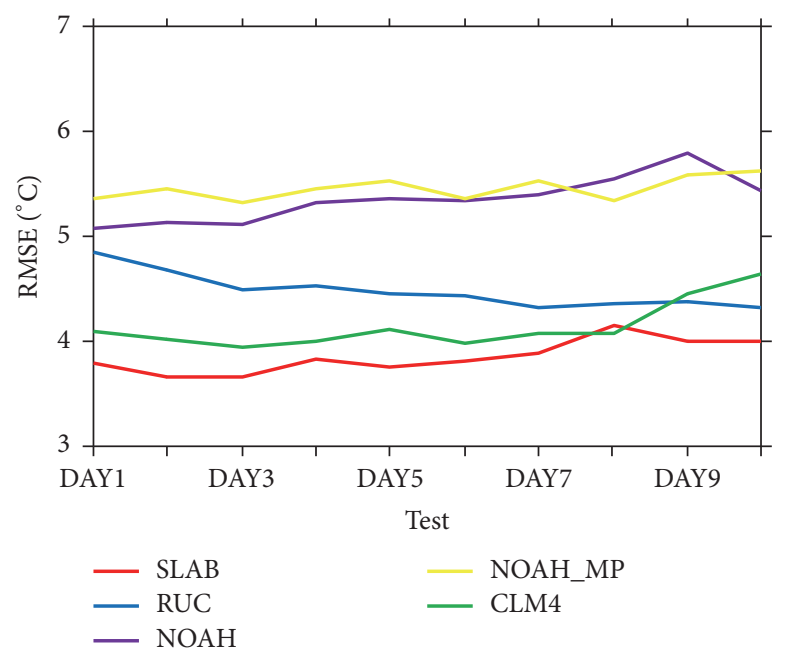

(c)

FIGURE 5: Assessment using the following measures: (a) TS, (b) BIAS, and (c) RMSE. 
scores. Whether in the short- or medium-range simulations, the errors associated with NOAH_MP and NOAH are the largest, and their BIAS (RMSE) values are all $-4^{\circ} \mathrm{C}\left(5.5^{\circ} \mathrm{C}\right)$ more or less. The BIAS values of RUC and SLAB are all between $-2^{\circ} \mathrm{C}$ and $-3^{\circ} \mathrm{C}$. The BIAS values of CLM4 are minimal (within $-1^{\circ} \mathrm{C}$ ), while the RMSE values associated with SLAB are minimal, about $4^{\circ} \mathrm{C}$. For the same simulation length, there are considerable differences in the errors produced by different LSSs. For example, the BIAS difference of CLM4-NOAH_MP is approximately $3.3^{\circ} \mathrm{C}$ in the DAY6 experiment.

For a given LSS, the errors can appear larger due to changes in simulation length. In terms of BIAS, CLM4 and RUC both show substantial changes with simulation length. Of those, CLM4 fluctuates up and down, and the maximum degree of change is more than $1^{\circ} \mathrm{C}$; RUC changes monotonically from $-3.1^{\circ} \mathrm{C}$ to $-2^{\circ} \mathrm{C}$; SLAB shows the smallest changes, which are relatively stable. In terms of their RMSE values, the rest of the LSSs show a slightly rising trend, of which the largest increase is exhibited by CLM4, which rises from $4.1^{\circ} \mathrm{C}$ to $4.6^{\circ} \mathrm{C}$; NOAH and NOAH_MP are generally similar and show increases of approximately $0.2^{\circ} \mathrm{C}$. On the other hand, RUC shows a slight decline of $0.52^{\circ} \mathrm{C}$.

Zeng et al. [14] found that the differences in the mediumrange errors associated with different LSSs were significantly larger than those in the short-term errors in East China. In this paper, the differences in the medium-range errors associated with different LSSs are essentially similar to those over the short term in the Sichuan-Chongqing area. Considering these errors, the SAT06 differences caused by the different LSSs are greater than the differences caused by different simulation lengths.

Based on the results of Student's $t$-test, the TS values, and the error analysis, CLM4 has the best simulation results for high temperatures, whereas NOAH and NOAH_MP are equivalent and the worst. Comparing RUC and SLAB, the TS of RUC is higher than that of SLAB, but the RMSE of RUC is slightly larger than that of SLAB, which indicates that neither of the two LSSs can achieve the best result in all aspects and each has its advantages and disadvantages.

\section{Explanation of Simulated Differences}

The above results show that the great differences in simulated SATs can be caused by different LSSs and different simulation lengths. These differences are mainly due to the influence of different LSSs on the surface energy and the errors caused by different simulation lengths. Therefore, we explain the differences in simulated high temperatures based on two aspects, the surface energy budget and soil characteristics. After that, we give the quantitative results of each mechanism.

4.1. Surface Energy Budget. The surface energy budget has a very important influence on SATs. In the model, the net energy fluxes from the surface are used for direct heating of the soil and causing the surface-soil temperature changes. These changes in surface-soil temperatures then lead to changes in the sensible heat flux (SHF), the latent heat flux
(LHF), and radiation energy, which can be transported to the atmosphere and affect the temperature in the bottom layer. The energy budget equation for the surface soil is given as follows:

$$
C_{s} \frac{\partial T}{\partial t}=R_{n}-(H+L E)-G .
$$

On the left side of the equation, $C_{s}$ and $\partial T / \partial t$ are the surface specific heat and temperature change, respectively. In the net energy flux on the right side of the equation, $R_{n}$ is the net radiation energy, $H$ is the SHF, $L E$ is the LHF, and $G$ is the soil heat flux.

From the surface net energy at 06 UTC averaged over simulated area $\mathrm{A} 3$ over different simulation lengths, it can be seen that NOAH and NOAH_MP are very close; however, the other LSSs estimate very different net energy values, and the biggest difference reaches $180 \mathrm{~W} \mathrm{~m}^{-2}$ (SLAB-RUC, DAY2) (Figure 6(a)). By comparing the variation of the average surface-soil temperature at 06 UTC with the simulation lengths, it can be found that although the net energy of SLAB is the largest, that of RUC is the minimum, but the simulated surface-soil temperatures show the opposite pattern (Figure 6(b)). That is, because the net energy is used to characterize the change rate of surface-soil temperature, positive values indicate that the surface-soil temperature is not the largest and can continue to increase. The net energy of SLAB (RUC) is large (small), which shows that the potential for temperature to increase is large (small). These results also show that 06 UTC may have not yet reached the highest temperatures within a day's time in the Sichuan-Chongqing area. Comparison of SAT06 and surface-soil temperature (Figures 3(j) and 6(b)) shows that the average SAT06 and the average surface-soil temperature simulated by each LSS have the same trend with simulation time. At the same time, the size relationship between SAT06 and surface-soil temperature is not consistent with different LSSs for the same simulation time (i.e., lower SAT does not correspond to lower surface-soil temperature). Therefore, the change in surfacesoil temperature caused by surface energy is very important to SAT, but other factors also affect SAT.

Given the differences between surface-soil temperature and SAT06 mentioned above, the differences in SLAB are the least, which is consistent with the minimum temperature lapse rate of SLAB in the surface layer in [14]. At the same time, the other LSSs show greater lapse rates.

SHF and LHF are two components of the energy flux and are the main distribution forms of surface net radiation. Simulation results show that SHF and LHF can show very large differences between LSSs (Figures 6(c) and 6(d)), mainly during the daytime ground heating stage (the highest value is approximately 2 times the minimum value; Figures 6(c) and 6(d)). It is worth noting that some LSSs-simulated SHF values "just" reach their maximum value at 06 UTC, and some have reached the maximum value by 05 UTC. Because the SHF directly heats the lower atmosphere, this further shows that different LSSs can not only cause differences in SAT06 but also be seen in that the time when the maximum temperature occurred can differ by more than $1 \mathrm{~h}$. 

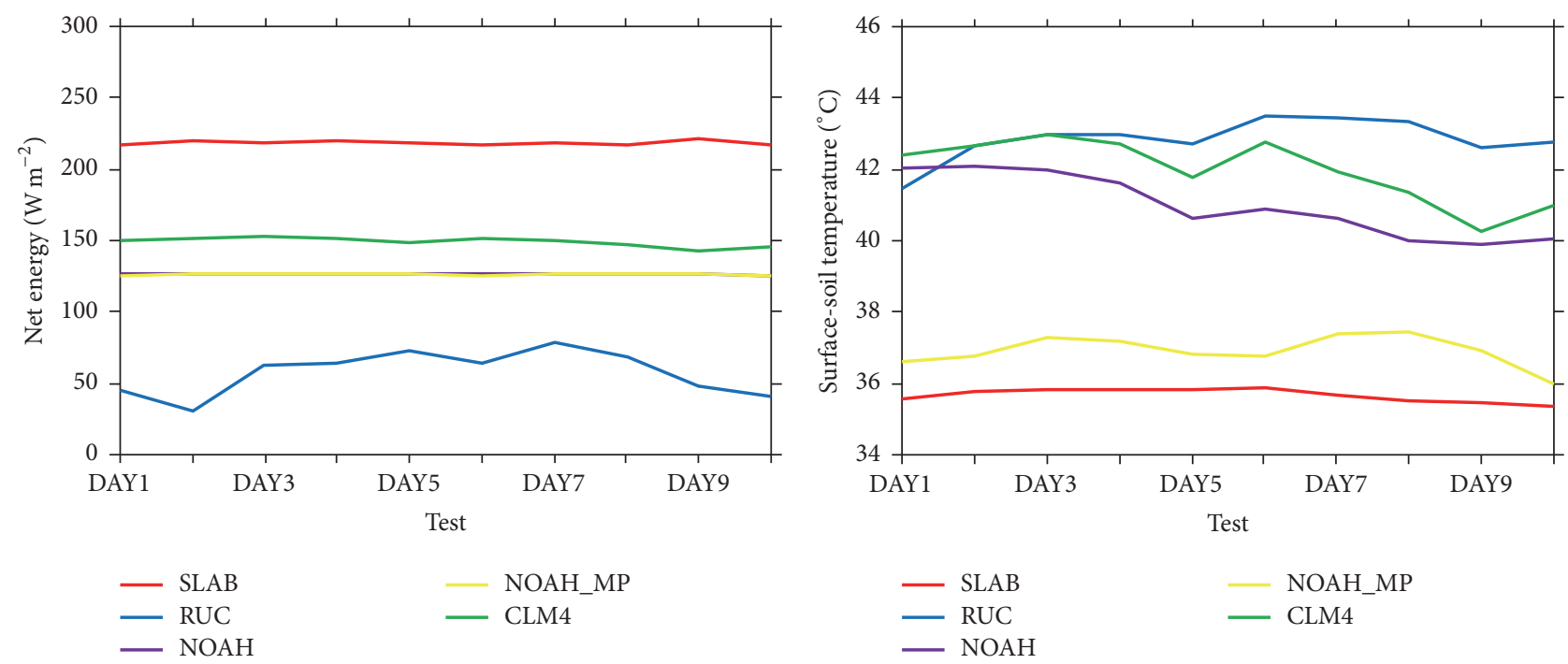

(a) 06 UTC averages
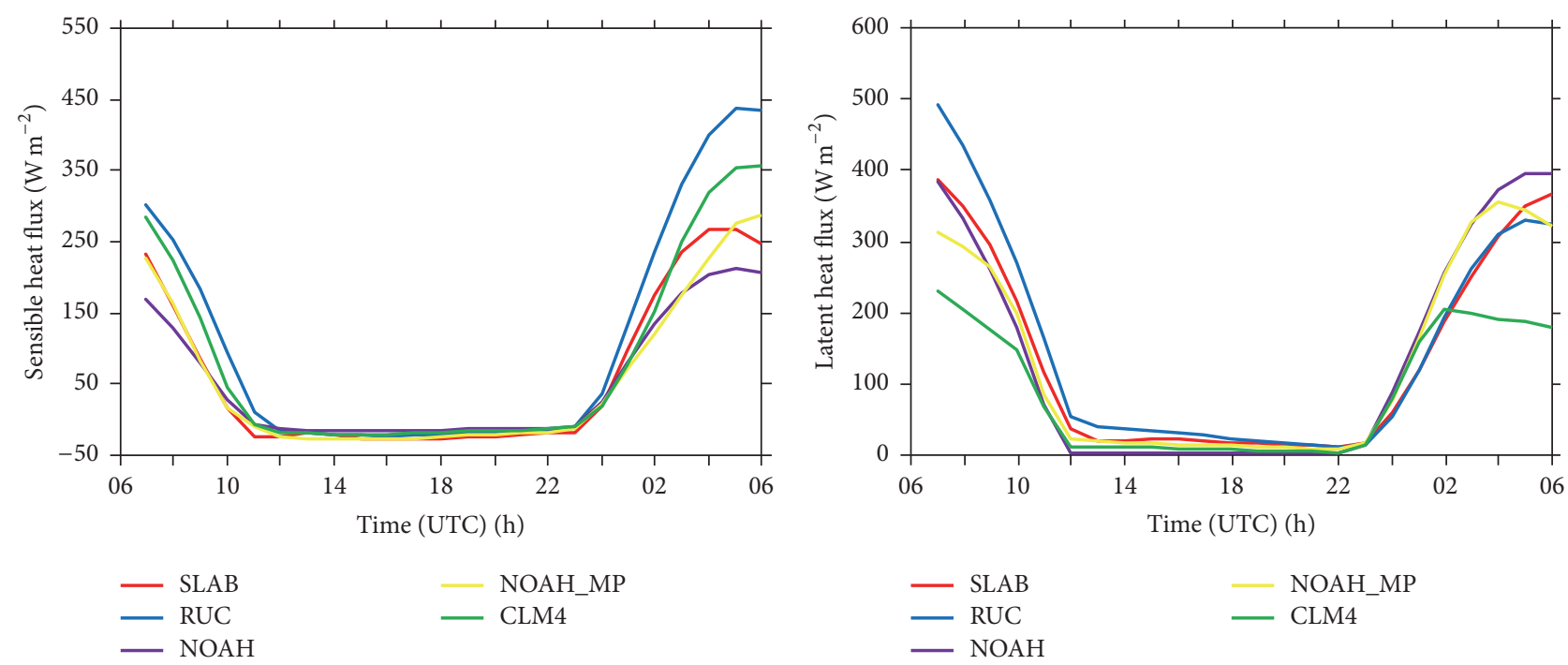

(c) DAY1 diurnal variation averages

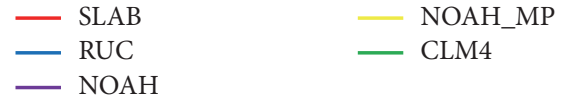

(d) DAY1 diurnal variation averages
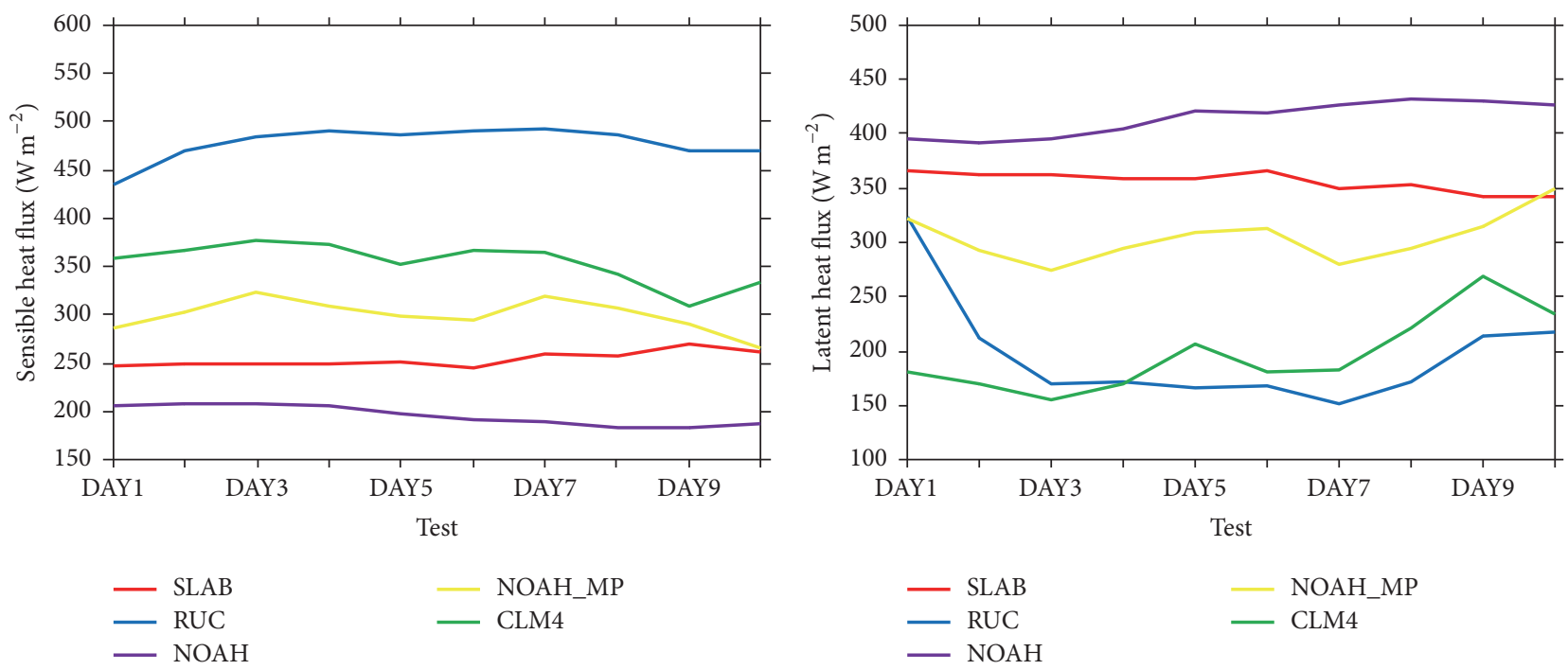

(e) 06 UTC averages

(f) 06 UTC averages

Figure 6: Continued. 


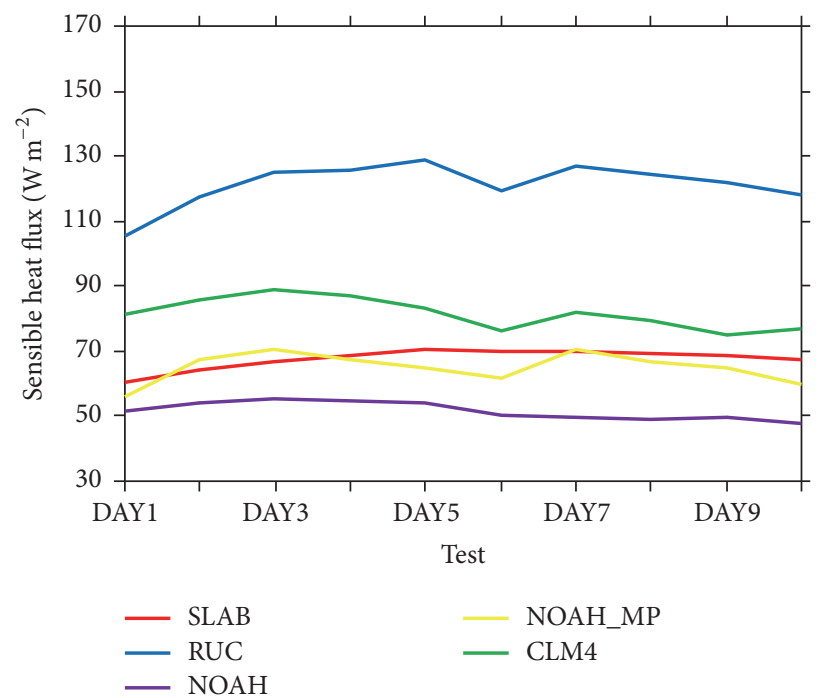

(g) Means derived from hourly values UTC averages

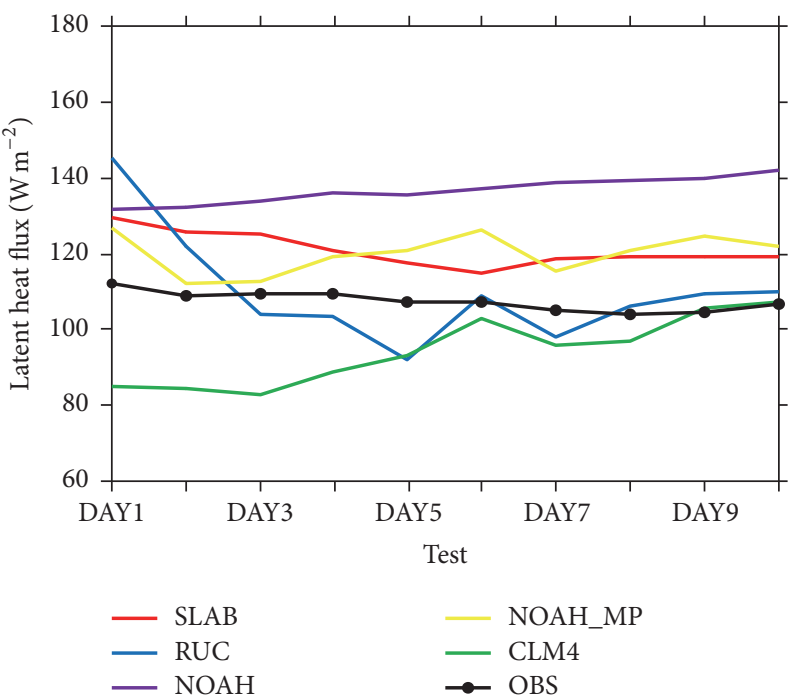

(h) Means derived from hourly values UTC averages

Figure 6: The simulated net energy at 06 UTC on August 10 (a), (b) the same as Figure 3(j) but for surface-soil temperature, (c) area A3averaged DAY1 diurnal SAT variations in SHF, (d) area A3-averaged DAY1 diurnal SAT variations in LHF, (e) the same as (a) but for SHF, (f) the same as (a) but for LHF, (g) the same as (e) but from the hourly WRF outputs, (h) the same as (f) but from the hourly WRF outputs, including observation-based data from daily evaporations by Miralles et al. [13].

The systematic differences of simulated SHF and LHF values, as well as the good correspondence between the changes in hourly quantities and the instantaneous quantities, are also observed here (Figures 6(e), 6(g), 6(f), and 6(h)). This shows that although SAT06 is the cumulative result from the long simulation period, the analysis of instantaneous quantities at 06 UTC can also reflect the physical mechanism. At the same time, the simulated results of LHF are close to the measured data (Figure 6(h)), but the differences in SHF are greater due to the different LSSs (Figure 6(g)).

A very unusual phenomenon is found through this comparison in that, unlike the other 4 LSSs, only the SLABsimulated SHF in the area of high temperatures is lower than that in the surrounding area, while the simulated LHF is higher in the area of high temperatures than the surrounding area (e.g., Figure 7). As the SHF directly heats the lower atmosphere, it is noted that SLAB still simulates the area of high temperatures (Figure 2(a)). This may mean that (1) the SLAB LSS is too simple to accurately represent the land surface physical process or (2) the transport of SHF sometimes does not have a major impact on the distribution of SATs (other aspects of the impact are discussed in Section 4.3). In fact, this weak correlation between SAT06 and SHF is also reflected in other experiments (e.g., RUC and CLM4; Figures $3(j)$ and 6(e)). These results are different from [14], in which temperature and SHF show a very good positive correlation, which may be closely related to the unique geographical features and the prevailing weather systems in the SichuanChongqing area.

4.2. Soil Moisture and Stratification. SM is a very important physical quantity affecting land-air interactions. Its initial values and changes have an important influence on the simulated SAT06 values [11].
Figure 8 shows the initial SM values averaged over area A3 simulated by 4 of the LSSs and FNL data in each experiment and the average SM during the whole period in each experiment using the hourly simulation results. It can be seen that large differences appear in the trend between the surface SM and the deep layer SM with simulation lengths. The initial surface SM values simulated by RUC are relatively lower than those of FNL, with a mean difference of $0.1 \mathrm{~m}^{3} \mathrm{~m}^{-3}$, and show a change that contrasts with its deep SM, so there is a greater inconsistency (Figures 8(a) and 8(i)), which shows that RUC seriously underestimates the actual SM. In terms of size and trend, the NOAH-simulated initial SM values are the most consistent with the FNL data in each layer and at a depth of $1 \mathrm{~m}$ (Figures 8(c) and 8(i)). In fact, the FNL data are obtained through the model assimilation coupling with NOAH. The above results reflect the performance of soil initialization for the different LSSs and their systematic characteristics related to their time evolution. Combined with the LSSs-simulated high-temperature performances in the experiments (Figure 3(j)), these results show that even if some of the LSSs-simulated initial SM values are more consistent with the driving data, the simulated high temperatures are not necessarily good.

NOAH, NOAH_MP, and CLM4 also simulate the changing characteristics of SM more accurately (Figure $8(\mathrm{j})$ ); that is, the average SM increased slowly with increasing simulation time. In addition, NOAH also showed a greater upward trend than NOAH_MP and CLM4 (Figures 8(d), 8(f), and $8(\mathrm{~h})$ ); however, NOAH simulates more evaporation than NOAH_MP and CLM4, and the evaporation increases with increasing simulation length (Figure 6(h)). In fact, this is because NOAH simulates greater average daily precipitation in each experiment that increases with increasing simulation length, and the water that falls to the ground is constantly 


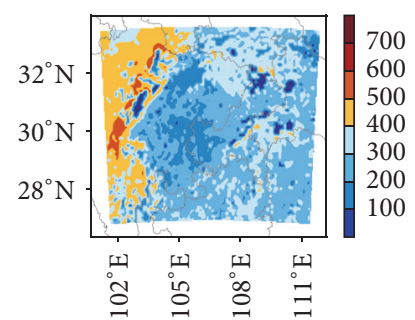

(a) SHF (SLAB DAY1)

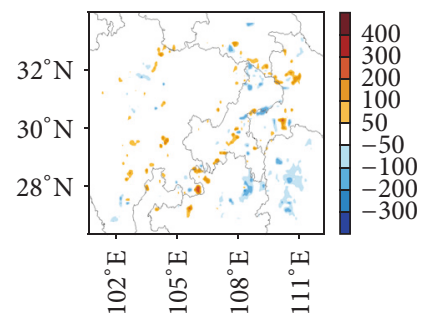

(e) DAY1-DAY5 (SLAB LHF)

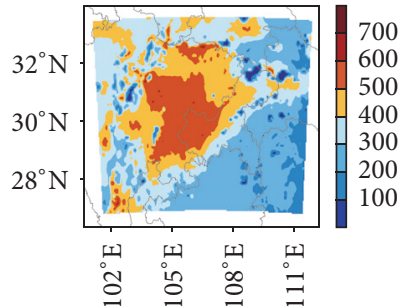

(b) SHF (RUC DAY1)

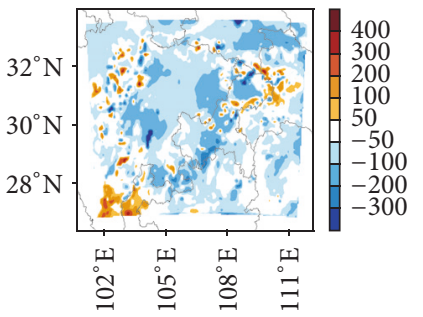

(f) DAY1-DAY10 (RUC SHF)

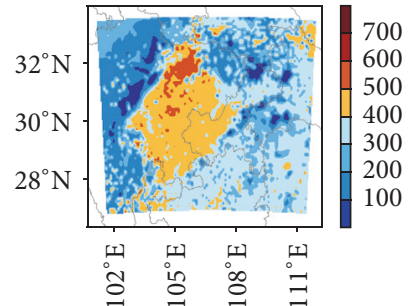

(c) LHF (SLAB DAY1)

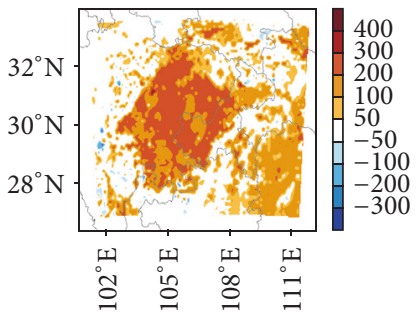

(g) CLM4-NOAH (DAY1 SHF)

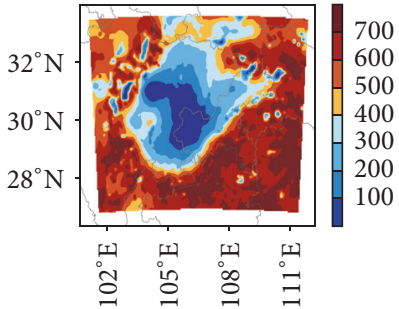

(d) LHF (RUC DAY1)

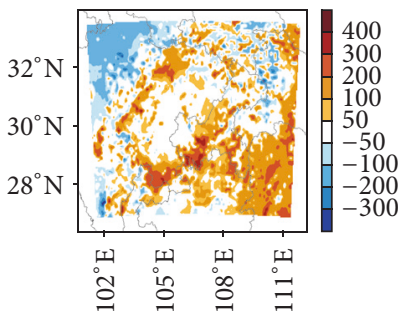

(h) CLM4-NOAH_MP (DAY4

FIGURE 7: Distributions of SHF and LHF at 06 UTC on August 10 simulated by the SLAB and RUC LSSs (a-d); distributions of SHF and LHF difference fields at 06 UTC on August 10 with the different LSSs and simulation lengths (e-h) (units: $\mathrm{W} \mathrm{m}^{-2}$ ).

added to the soil (Figure $8(\mathrm{k})$ ). The greater amount of precipitation and the greater SM values are also important reasons for the lower temperature simulated by NOAH (Figure 3(j)). Although the daily average precipitation simulated by RUC also increases with simulation length (Figure $8(\mathrm{k})$ ), its average SM is slightly decreased (Figure 8(b)). Taking into account the fact that its evaporation is also declining (Figure 6(h)), the strong gravity drainage therefore acts as the main cause of the decline in the SM values simulated by RUC. It is this kind of action that leads to the reduction in SM and thus causes the enhancement of the SHF which eventually leads to increases in the SATs simulated by RUC and a rise of $1^{\circ} \mathrm{C}$ in the medium term compared to the short term (Figure 3(j)).

In addition, high-temperature effects are considered from the point of view of soil layers. We note that PX, which has 2 soil layers, is not able to simulate high temperatures. It will be found that the high-temperature effect decreases in accordance with the order of CLM4 (10 layers), RUC (6 layers), SLAB (5 layers), NOAH (4 layers), NOAH_MP (4 layers), and PX (2 layers). That is, the greater the number of soil layers an LSS has is, the higher the simulated SATs are, and the closer they lie to the measured values. This is because the more the soil layers, the more precise the conduction processes within soil and the surface energy in terms of the internal parameters that represent those processes and the lower the likability of having large errors. Therefore, the possibility of a more accurate distribution of simulated $2 \mathrm{~m}$ temperatures is relatively high [23].

4.3. Quantitative Analysis. To quantitatively analyze the contribution of each mechanism or factor to the temperature variations, the temperature change equation is as follows:

$$
\frac{\partial T}{\partial t}=-\left(u \frac{\partial T}{\partial x}+v \frac{\partial T}{\partial y}\right)-w\left(\gamma_{d}-\gamma\right)+Q
$$

The variables $u$ and $v$ represent the horizontal wind speeds with directions of $x$ and $y$, respectively, $w$ represents the vertical wind speed, $\gamma_{d}$ and $\gamma$ represent the adiabatic lapse rate and the temperature lapse rate, respectively, and $Q$ represents diabatic heating.

We set

$$
\begin{aligned}
T_{t} & =\int_{t} \frac{\partial T}{\partial t} \mathrm{~d} t \\
\mathrm{ADV} & =-\int_{t} V \cdot \nabla T \mathrm{~d} t \\
\mathrm{CON} & =-\int_{t} w\left(\gamma_{d}-\gamma\right) \mathrm{d} t . \\
H_{t} & =\int_{t} \mathrm{Q} \mathrm{d} t .
\end{aligned}
$$

These represent integral terms for local temperature changes, advection, convection (subsidence warming), and diabatic heating, respectively. Therefore, (2) can be rewritten as

$$
T_{t}=\mathrm{ADV}+\mathrm{CON}+H_{t}
$$

Because there are few $2 \mathrm{~m}$ meteorological quantities in the model outputs, we choose the lowest model layer (approximately $30 \mathrm{~m}$ ) to calculate the contribution from each factor to the temperature variation from 00 UTC to 06 UTC on behalf of the heating process, in order to make a quantitative analysis of SAT changes. Figures 9(a)-9(d) give the hourly change rates of $T_{t}, H_{t}, \mathrm{CON}$, and ADV. It can be seen that $T_{t}$ of RUC (NOAH) is the largest (smallest) and the 10-experiment mean $T_{t}$ is $1.24^{\circ} \mathrm{Ch}^{-1}\left(0.95^{\circ} \mathrm{Ch}^{-1}\right)$, and these values are reduced in the medium term relative to the short term for all the LSSs (Figure 9(a)). The $H_{t}$ 




(a) RUC-Initial

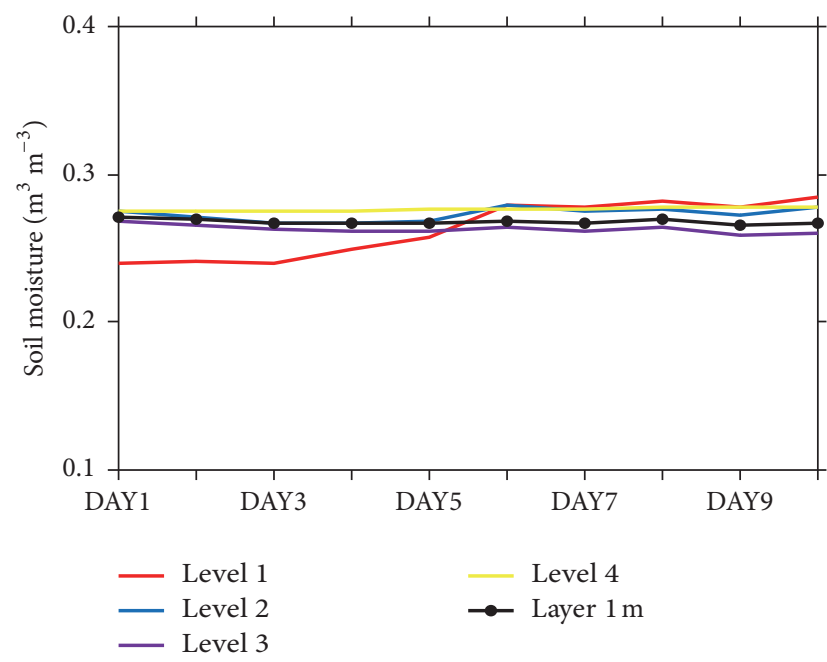

(c) NOAH-Initial

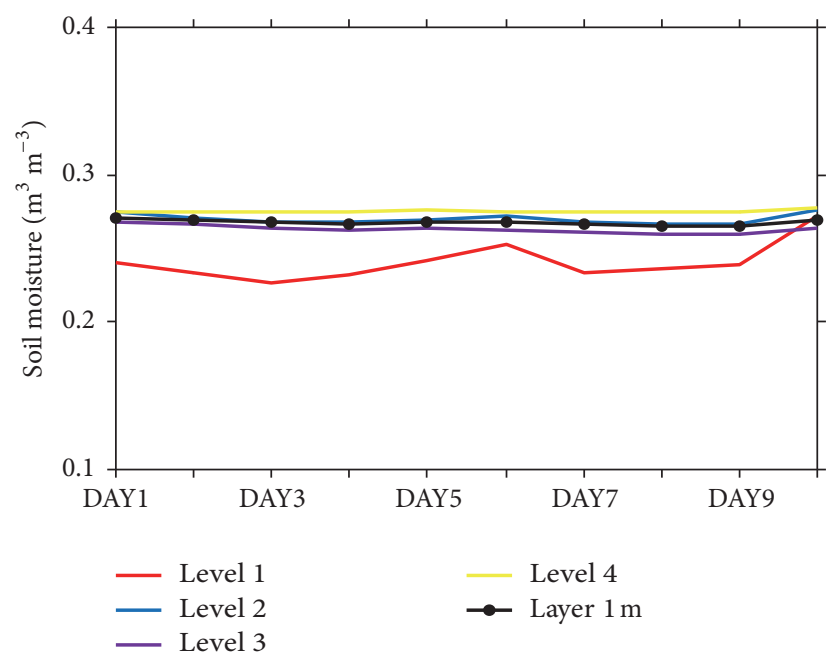

(e) NOAH_MP-Initial

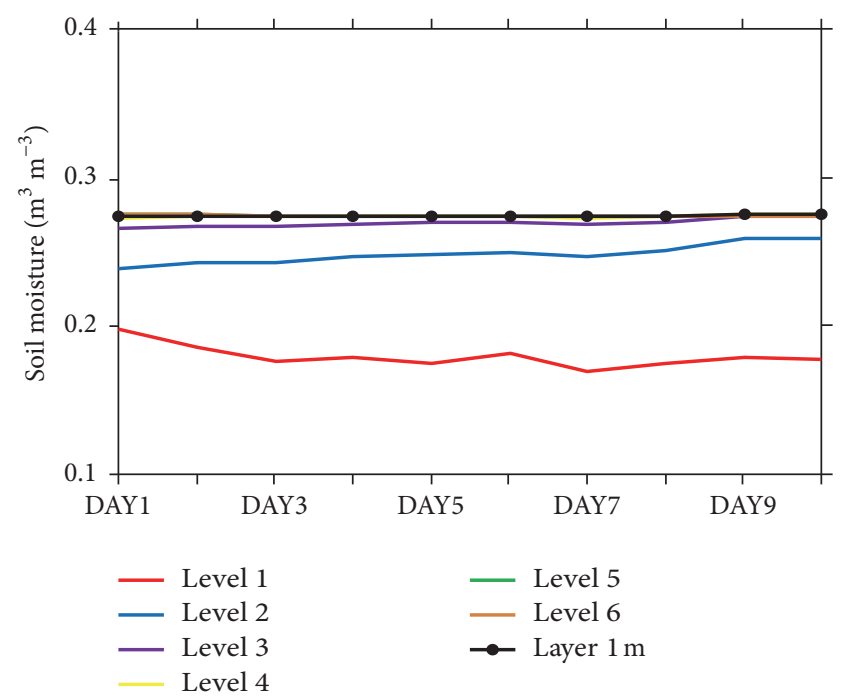

(b) RUC-Temporally Averaged

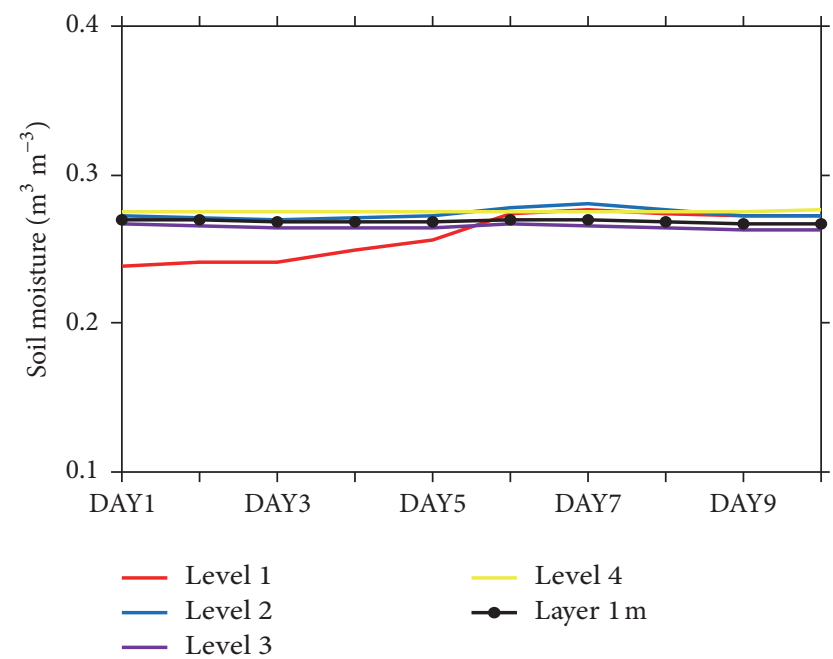

(d) NOAH-Temporally Averaged

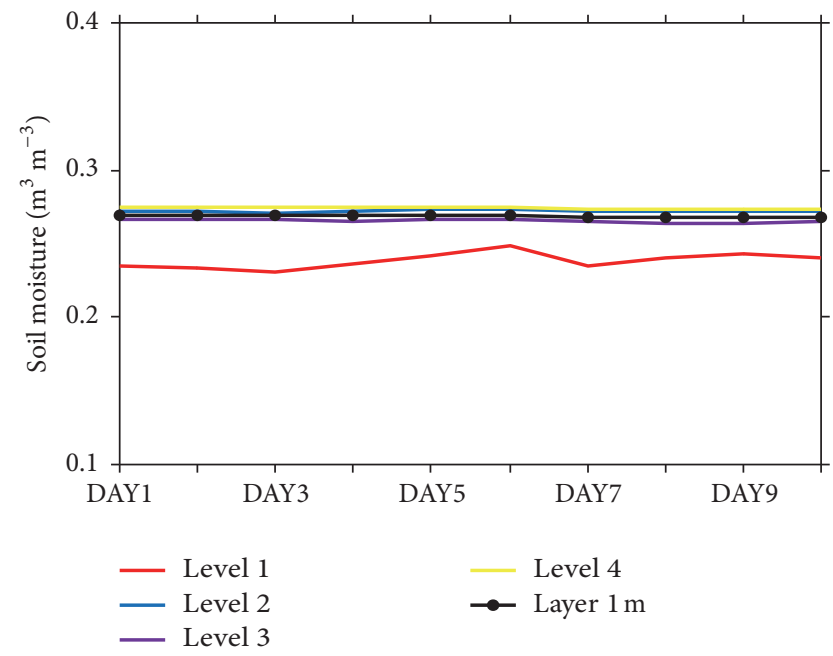

(f) NOAH_MP-Temporally Averaged

FIgure 8: Continued. 




(g) CLM4-Initial



(i) FNL-Initial

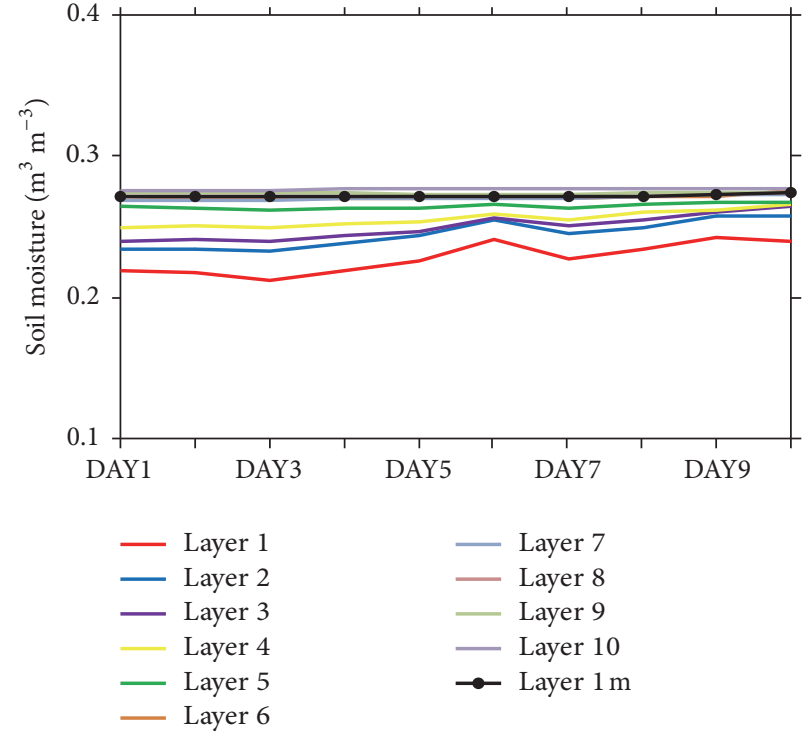

(h) CLM4-Temporally Averaged

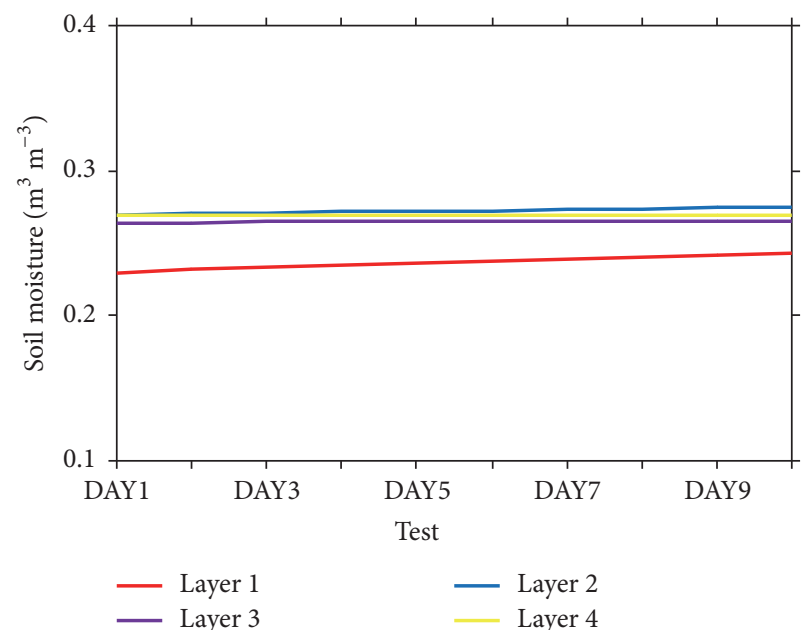

(j) FNL-Temporally Averaged

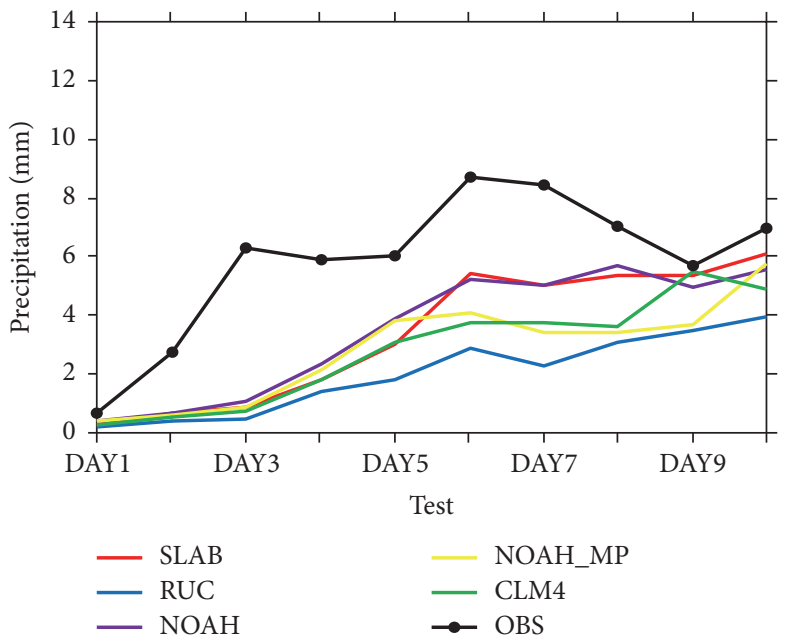

(k) Precipitation-Temporally Averaged

FIGURE 8: (a, c, e, g, and i) Domain A3-averaged initial soil moisture contents (unit: $\mathrm{m}^{3} \mathrm{~m}^{-3}$ ) and (b, d, f, h, and j) soil moisture variations (unit: $\mathrm{m}^{3} \mathrm{~m}^{-3}$ ) for the LSSs over time for the following soil layers: RUC (Level 1: $0 \mathrm{~cm}$; Level 2: $5 \mathrm{~cm}$; Level 3: $20 \mathrm{~cm}$; Level 4: $40 \mathrm{~cm}$; Level 5: $160 \mathrm{~cm}$; Level 6: $300 \mathrm{~cm}$ ), NOAH and NOAH_MP (Layer 1: 0-10 cm; Layer 2: 10-40 cm; Layer 3: 40-100 cm; Layer 4: 100-200 cm), CLM4 (Layer 1: 0-1.75 cm; Layer 2: 1.75-4.51 cm; Layer 3: 4.51-9.06 cm; Layer 4: 9.06-16.56 cm; Layer 5: 16.56-28.92 cm; Layer 6: 28.92-49.3 cm; Layer 7: 49.3-82.9 cm; Layer 8: 82.9-138.29 cm; Layer 9: 138.29-229.62 cm; Layer 10: 229.62-343.32 cm), and FNL (Layer 1: 0-10 cm; Layer 2: 10-40 cm; Layer 3: 40-100 cm; Layer 4: 100-200 cm); (k) daily average precipitation variations for the LSSs and observations over time (unit: mm). 


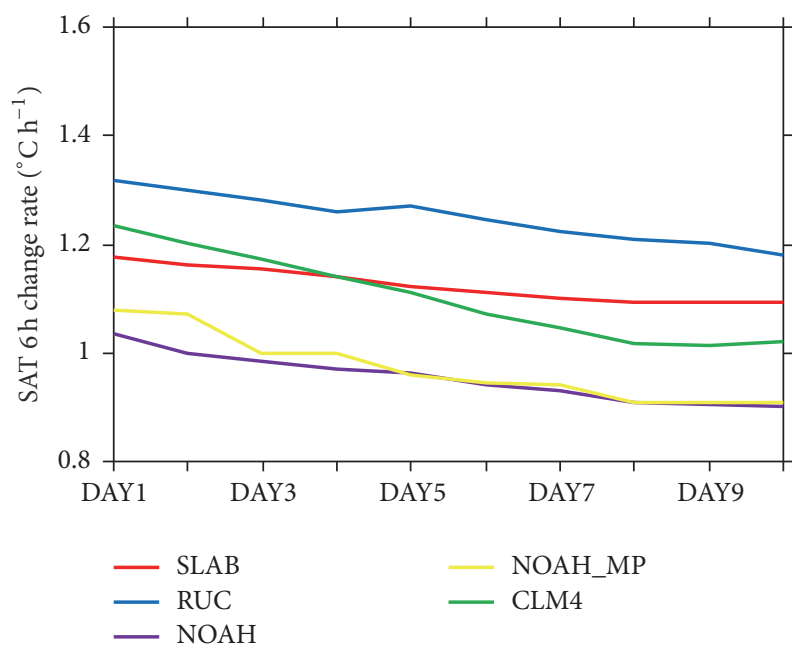

(a) The $T_{t}$ term

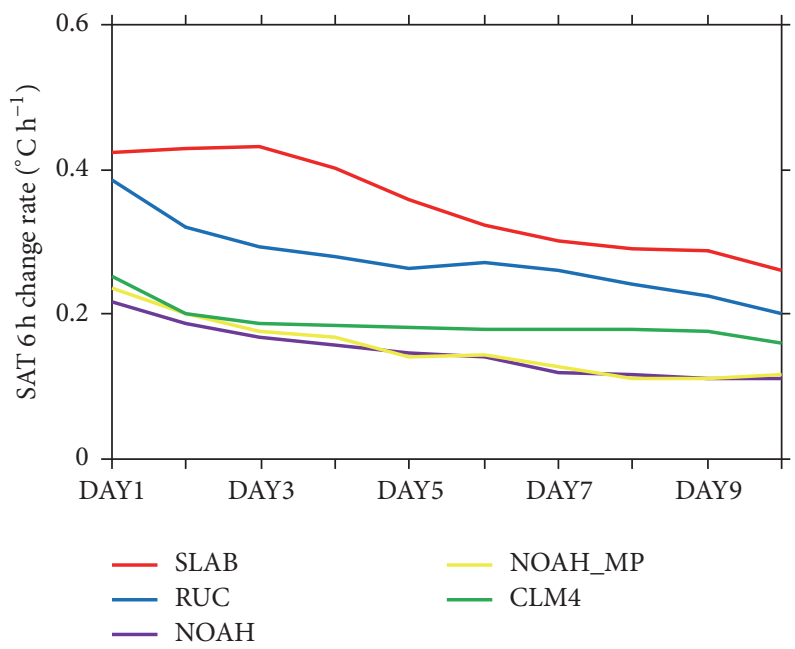

(c) The CON term

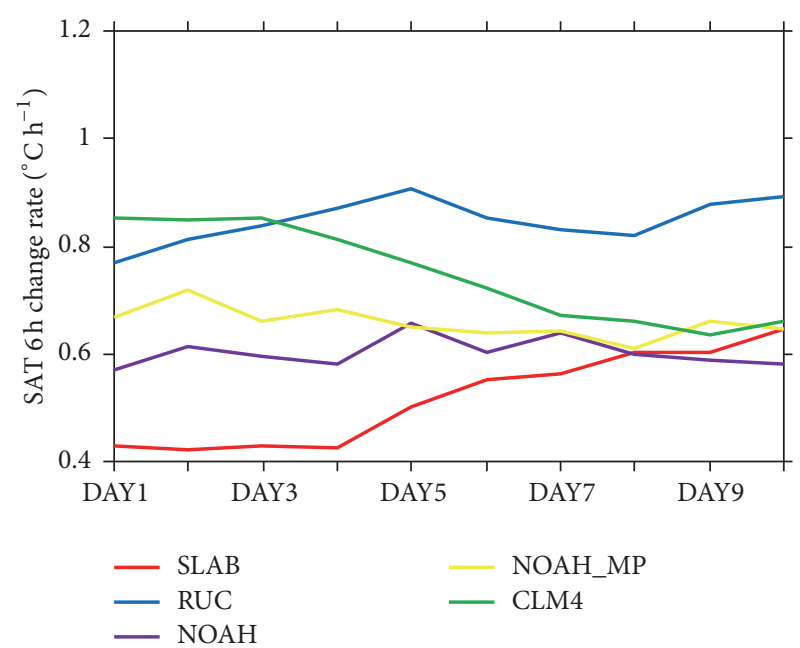

(b) The $H_{t}$ term

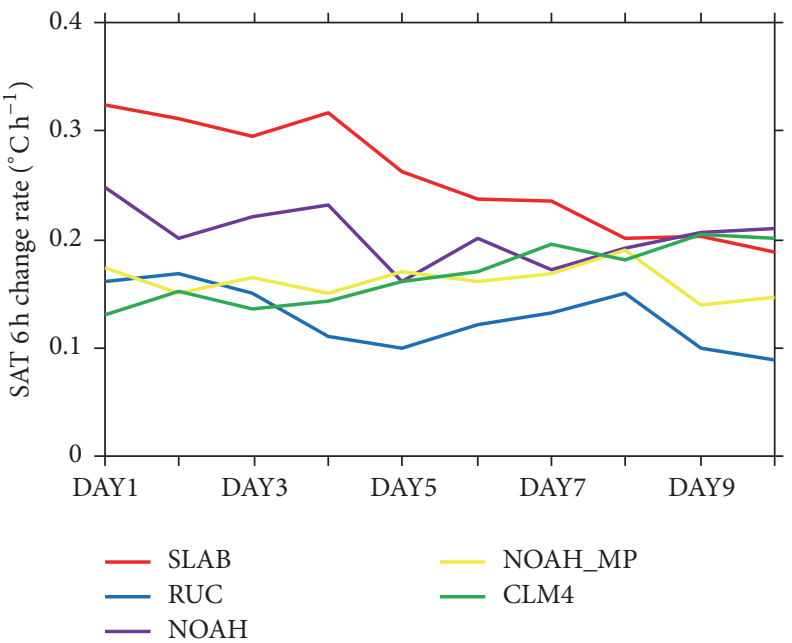

(d) The ADV term

FIGURE 9: Domain A3-averaged change rates of SAT (unit: ${ }^{\circ} \mathrm{Ch}^{-1}$ ) for the $T_{t}(\mathrm{a}), H_{t}$ (b), CON (c), and ADV (d) terms.

values simulated by all the LSSs are larger than CON and ADV. In the 10-experiment mean, $H_{t}$ of CLM4 (SLAB) is the largest (smallest) at $0.75^{\circ} \mathrm{Ch}^{-1}\left(0.52^{\circ} \mathrm{Ch}^{-1}\right)$, which makes up $68 \%(46.2 \%)$ of $T_{t}$ (Figure $9(\mathrm{~b})$ ), which is related to the inconsistency between SLAB-simulated SATs and SHF (Figures 7(a) and 7(c)).

The mean CON of SLAB (NOAH) is the largest (smallest) at $0.35^{\circ} \mathrm{Ch}^{-1}\left(0.15^{\circ} \mathrm{Ch}^{-1}\right)$, the proportion of which in $T_{t}$ is 29.6\% (15.4\%) (Figure 9(c)). All LSSs-simulated ADV values are positive, representing the role of warm advection, the largest (smallest) proportion of which in $T_{t}$ is simulated by SLAB (RUC) at $22.6 \%(10.3 \%)$ (Figure 9(d)). Thus, SATs in the heating process mainly rely on the role of diabatic heating; the contribution from subsidence warming is secondary and that of advection is small but still cannot be ignored in simulating summer extreme high temperatures in the Sichuan-Chongqing area.

In Section 4.1, above, the SHF simulated by CLM4 is lower than that of RUC, but SAT06 is just the opposite before DAY8. The reason is more complicated. First, the quantitative analysis results show that the SHF of CLM4 is low, but the diabatic heating's contribution to SAT change is higher, especially before DAY3, which indicates that there is little consistency between the SHF and diabatic heating. Diabatic heating represents a net energy flux of a certain thickness of air near the ground, while SHF represents only one level of energy and is a part of diabatic heating. Second, the advection effect on SAT change of CLM4 is higher than that of RUC. Third, the boundary layer and SM come from observations based on the underlying surface, which has a simple and homogeneous level distribution, so the parameterization schemes cannot completely simulate the process of land-air exchange in the Sichuan-Chongqing area.

\section{Conclusions}

The Sichuan-Chongqing area is known for its extremely complex basin terrain and extremely hot weather in summer. To 
study the impact of LSS choice on a simulated heatwave event, the mesoscale model WRF 3.6.1 and six LSSs (i.e., SLAB, RUC, PX, NOAH, NOAH_MP, and CLM4) are selected, and other parameter settings are taken to be the same. A total of 60 experiments covering 1 to 10 days are designed to simulate the high-temperature weather on August 10 of 2006 in the Sichuan-Chongqing area.

The results show that the parameterized algorithm PX does not apply in the Sichuan-Chongqing area, and PX completely failed to simulate the high-temperature distribution in both the short- and the medium-range experiments. This may be related to the serious deficiencies in the internal soil parameterization (such as its stratification and internal water flux calculation) of the PX LSS. The other 5 LSSs (the following discussion only treats these 5 LSSs) successfully simulated the distribution of high temperatures over the short and medium term. The simulation of high temperatures is also sensitive to the different LSSs. The specific performance of each LSS has distinct characteristics. For short-term simulations of 1-2 days, the hightemperature distribution of CLM4 is the most consistent with the measurements; RUC and SLAB follow; NOAH is slightly better than NOAH_MP, consistent with their TSs (threat scores). According to the error analysis, the BIAS of CLM4 is the smallest and the RMSE of SLAB is the smallest, the BIAS and RMSE of NOAH_MP are the largest, and the difference in BIAS between CLM4 and NOAH_MP is $2.5^{\circ} \mathrm{C}$. For medium-range simulations covering 3-10 days, the performances of CLM4, RUC, and SLAB are consistent with their performances in short-term simulations. The CLM4simulated high-temperature strength significantly reduced after DAY8 and became lower than that of RUC, but the distribution produced by CLM4 is still the best. There is a big difference between NOAH and NOAH_MP compared with their short-term simulations; that is, the distribution and TS of NOAH_MP are better than those of NOAH in the medium term, but the BIAS and RMSE of NOAH_MP are still the biggest on the whole; the difference in the BIAS between different LSSs can reach up to $3^{\circ} \mathrm{C}$ in the mediumrange simulations.

High temperatures simulated by the same LSS are also sensitive to the simulation length. The TS values of RUC show steady growth in experiments with lengths of 1-10 days and the error also diminishes; the BIAS declined by $1.2^{\circ} \mathrm{C}$ from DAY1 to Day10. The TS of CLM4 is relatively stable, and the growth of BIAS is more obvious; the maximum span of the BIAS is from $-0.9^{\circ} \mathrm{C}$ (DAY6) to $-2.2^{\circ} \mathrm{C}$ (DAY9). The TS values of the other three LSSs show significant decreasing trends, and the reduction in the magnitude of NOAH is the largest, which shows a reduction from 0.31 to 0.11 . In terms of changes in the error, the BIAS of SLAB is relatively stable at around $-2.5^{\circ} \mathrm{C}$; although the BIAS values of NOAH and NOAH_MP do not show a large rise, the rising trend is obvious. Given the meaning of TS, BIAS, and RMSE, the high-temperature difference caused by the different LSSs is greater than that caused by the simulation lengths.

In general, the differences between the above simulated temperatures are mainly caused by different simulated surface fluxes, and the high temperatures and SHFs are generally positively correlated. However, for the extremely complex distribution of topography and vegetation in the SichuanChongqing area, although the SHF of CLM4 is smaller than that of RUC, the simulated temperature is higher, which is due to the inconsistency between SHF and diabatic heating in the near surface air as well as other possible complex physical factors. The maximum average contribution of diabatic heating for SAT changes in the heating process during the daytime is $68 \%$ (CLM4), and the minimum is $46.2 \%$ (SLAB).

The largest (smallest) proportion of subsidence warming in the SAT change is $29.6 \%(15.4 \%)$, whereas the largest (smallest) proportion of warm advection in the SAT change is SLAB (RUC) of $22.6 \%(10.3 \%)$. This result shows that the effect of subsidence warming is secondary. The effect of advection is small but still cannot be ignored in simulating the daytime heating process in the Sichuan-Chongqing area.

The change of SM may have an important influence on simulations. RUC still exhibits defects in terms of the initialized SM values, which are seriously low. Although the initial SM values of NOAH are consistent with the driving data, the effect in terms of the simulated high temperature is not good. In addition to the RUC, the simulated SM of the other LSSs is substantially increased with increasing simulation length (generally consistent with the FNL data). The main reason is because all the LSSs simulate precipitation in the SichuanChongqing area (NOAH is the largest). The larger SM values lead to a smaller SHF, which causes a decrease in temperature with increasing simulation length. While in the case that RUC also simulated increasing precipitation, the strong gravity drainage still leads to a decline in SM, and thus the simulated SAT06 is rising.

The simulation results of the LSSs in different soil layers showed that the greater the number of layers, the better the high-temperature effect. This is because the greater the number of soil layers is, the more precise the calculation of the internal moisture and energy fluxes will be, resulting in a greater ability to simulate the diffusion characteristics of the physical properties, so the simulation error is smaller. This proves the importance of soil layers in the simulation of high temperatures.

Although these simulations were only for the hightemperature weather that occurred on August 10, 2006, a series of short- and medium-range experiments also exhibit the system characteristics of high temperatures simulated by different LSSs. These are very meaningful for us to characterize model performance and to adopt different parameterization schemes for different purposes.

\section{Conflicts of Interest}

The authors declare that there are no conflicts of interest regarding the publication of this paper.

\section{Acknowledgments}

This work was financially funded by National Natural Science Foundation of China (Grant nos. 41675007 and 41275012). 


\section{References}

[1] C. W. Team, R. K. Pachauri, and L. A. Meyer, "Climate change 2014: synthesis report. Contribution of Working Groups I, II and III to the Fifth Assessment Report of the Intergovernmental Panel on Climate Change," Journal of Romance Studies, vol. 4, no. 2, pp. 85-88, 2014.

[2] J. A. Patz, D. Campbell-Lendrum, T. Holloway, and J. A. Foley, "Impact of regional climate change on human health," Nature, vol. 438, no. 7066, pp. 310-317, 2005.

[3] P. A. Stott, D. A. Stone, and M. R. Allen, "Human contribution to the European heatwave of 2003," Nature, vol. 432, no. 7017, pp. 610-614, 2004.

[4] L. X. Mao, S. Qian, Y. Y. Hou, and C. S. Li, "Study on the meteorologically-driven ecological monitoring and assessment of high temperature and drought of Sichuan-Chongqing area in summer 2006," Meteorological, vol. 33, no. 3, pp. 83-88, 2007.

[5] J. Shi, Y. H. Ding, and L. L. Cui, "Climatic characteristics of extreme maximum temperature in East China and its causes," Chinese Journal of Atmospheric Sciences, vol. 33, no. 2, pp. 347358, 2009.

[6] L. H. Chen, L. Zhou, J. T. Dang, Y. Hu, and F. Sheng, "Research on physical mechanism of summer severe climatic high temperature and drought disasters in Sichuan and Chongqing area in 2006," Meteorological, vol. 36, no. 5, pp. 85-91, 2010.

[7] R. Avissar and R. A. Pielke, "The impact of plant stomatal control on mesoscale atmospheric circulations," Agricultural and Forest Meteorology, vol. 54, no. 2-4, pp. 353-372, 1991.

[8] P. J. Sellers, R. E. Dickinson, D. A. Randall et al., "Modeling the exchanges of energy, water, and carbon between continents and the atmosphere," Science, vol. 275, no. 5299, pp. 502-509, 1997.

[9] J. B. Basara, H. G. Basara, B. G. Illston, and K. C. Crawford, “The impact of the urban heat island during an intense heat wave in oklahoma city," Advances in Meteorology, vol. 2010, Article ID 230365, 10 pages, 2010.

[10] N. Wolfson, R. Atlas, and Y. C. Sud, "Numerical experiments related to the summer 1980 U.S. heat wave," Monthly Weather Review, vol. 115, no. 7, pp. 1345-1357, 1987.

[11] E. M. Fischer, S. I. Seneviratne, P. L. Vidale, D. Lüthi, and C. Schär, "Soil moisture-atmosphere interactions during the 2003 European summer heat wave," Journal of Climate, vol. 20, no. 20, pp. 5081-5099, 2007.

[12] X. M. Zeng, Z. H. Wu, S. Y. Xiong, S. Song, Y. Q. Zheng, and H. Q. Liu, "Sensitivity of simulated short-range high-temperature weather to land surface schemes by WRF", Science China Earth Sciences, vol. 54, no. 4, pp. 581-590, 2011.

[13] D. G. Miralles, A. J. Teuling, C. C. Van Heerwaarden, and J. V.G. De Arellano, "Mega-heatwave temperatures due to combined soil desiccation and atmospheric heat accumulation," Nature Geoscience, vol. 7, no. 5, pp. 345-349, 2014.

[14] X.-M. Zeng, N. Wang, Y. Wang et al., "WRF-simulated sensitivity to land surface schemes in short and medium ranges for a high-temperature event in East China: a comparative study," Journal of Advances in Modeling Earth Systems, vol. 7, no. 3, pp. 1305-1325, 2015.

[15] H. Zhang, A. Henderson-Sellers, A. J. Pitman, J. L. McGregor, C. E. Desborough, and J. J. Katzfey, "Limited-area model sensitivity to the complexity of representation of the land surface energy balance," Journal of Climate, vol. 14, no. 19, pp. 3965-3986, 2001.

[16] W. C. Skamarock, J. P. Klemp, J. Dudhia et al., "A description of the advanced research WRF version 3," Tech. Rep. NCAR/TN-475+STR, NCAR Technical Note, 2008.
[17] J. Dudhia, "A multi-layer soil temperature model for MM5," in Proceedings of the 6th PSU/NCAR Mesoscale Model Users' Workshop, pp. 49-50, Boulder, Colo, USA, July 1996.

[18] T. G. Smirnova, J. M. Brown, S. G. Benjamin, and D. Kim, "Parameterization of cold-season processes in the MAPS landsurface scheme," Journal of Geophysical Research Atmospheres, vol. 105, no. 3, pp. 4077-4086, 2000.

[19] A. Xiu and J. E. Pleim, "Development of a land surface model. Part I: application in a mesoscale meteorological model," Journal of Applied Meteorology, vol. 40, no. 2, pp. 192-209, 2001.

[20] F. Chen and J. Dudhia, "Coupling an advanced land surfacehydrology model with the Penn State-NCAR MM5 modeling system. Part I: model implementation and sensitivity,' Monthly Weather Review, vol. 129, no. 4, pp. 569-585, 2001.

[21] G.-Y. Niu, Z.-L. Yang, K. E. Mitchell et al., "The community Noah land surface model with multiparameterization options (Noah-MP): 1. Model description and evaluation with localscale measurements," Journal of Geophysical Research Atmospheres, vol. 116, no. 12, pp. 1248-1256, 2011.

[22] K. W. Oleson, D. M. Lawrence, B. Gordon et al., "Technical description of version 4.0 of the Community Land Model (CLM)," Geophysical Research Letters, vol. 37, no. 7, pp. 256-265, 2010.

[23] J. R. Garratt, "Sensitivity of climate simulations to land-surface and atmospheric boundary-layer treatments-a review," Journal of Climate, vol. 6, no. 3, pp. 419-449, 1993. 

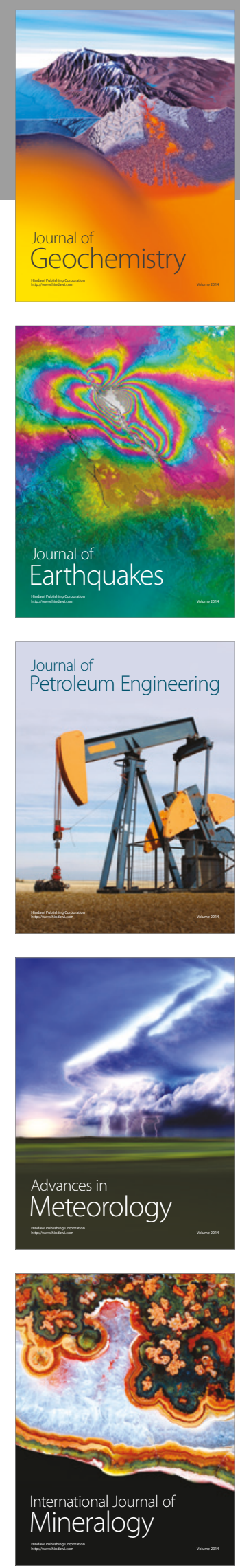
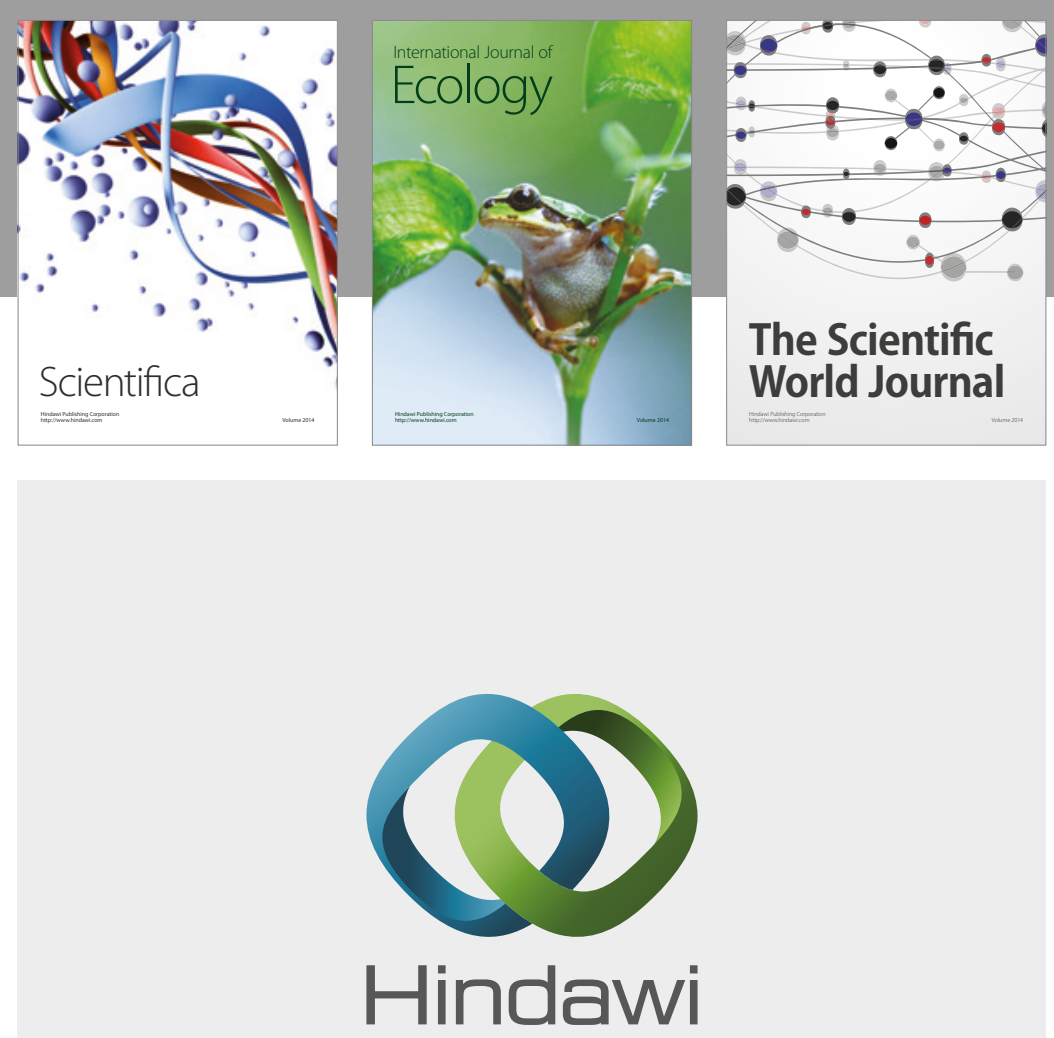

Submit your manuscripts at

https://www.hindawi.com
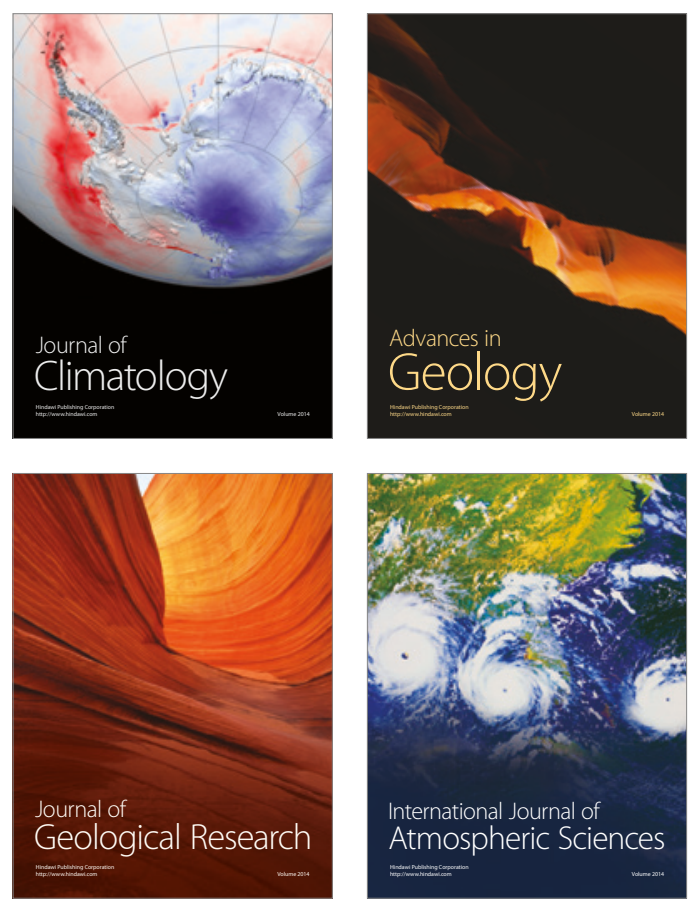

The Scientific

World Journal
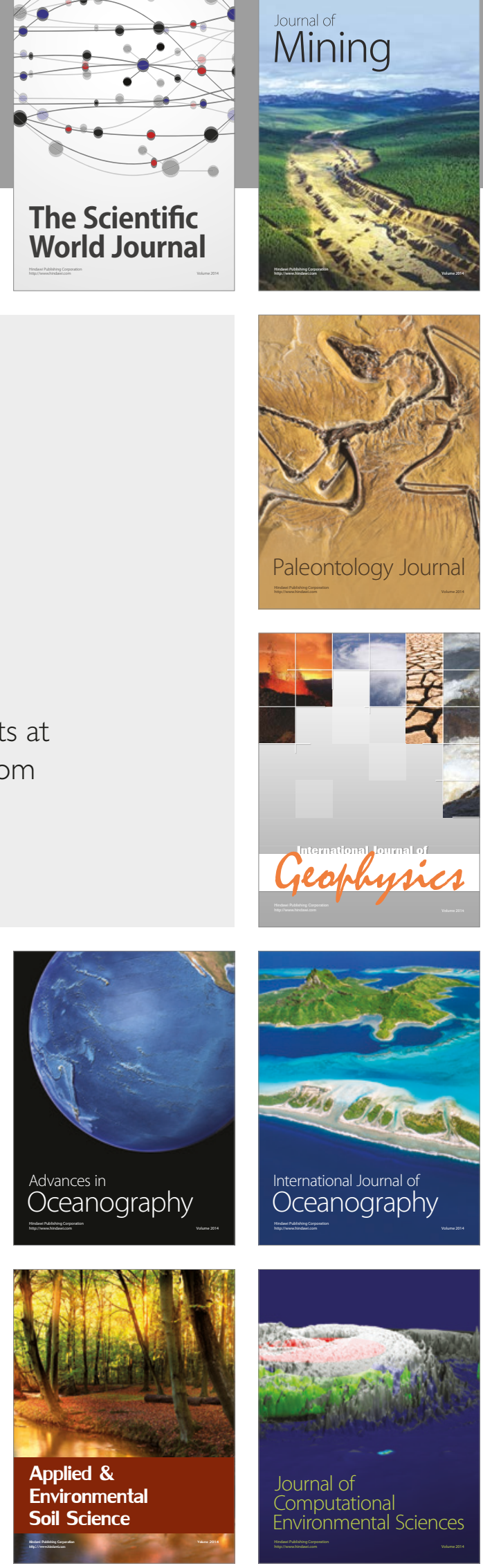